\title{
Typologische und technologische Untersuchungen der Saxe aus Überackern, Oberösterreich
}

\author{
Thomas Koch Waldner
}

\section{Zusammenfassung}

Das merowingerzeitliche Gräberfeld von Überackern kann anhand des vorliegenden Fundmaterials in das 7. und frühe 8. Jh. n. Chr. datiert werden. Die vier Saxe aus dem Gräberfeld wurden im Rahmen von Untersuchungen an frühmittelalterlichen Waffen aus Oberösterreich für typologische und technologische Analysen ausgewählt. Bei drei der vier Waffen aus Überackern handelt es sich um Langsaxe, die im letzten Drittel des 7. Jhs. auftreten und die typische Saxform des 8. Jhs. darstellen. Das älteste Exemplar zählt zur Gruppe der leichten Breitsaxe und datiert in die erste Hälfte des 7. Jhs.

Die Ergebnisse der metallografischen Analysen zeigen, dass die vier Saxe aus Überackern deutliche technologische Parallelen aufweisen. Sowohl beim leichten Breitsax als auch bei den drei Langsaxen wurde Schneidenmaterial aus hartem Stahl an Rückenmaterial aus weichem, jedoch zäherem Eisen angeschweißt. Diese Methode konnte bei sieben der neun untersuchten Saxe aus Oberösterreich festgestellt werden. Ein weiteres Verfahren, das als technologischer Standard des 7. und 8. Jhs. angesehen werden kann, ist das Härten von Klingen durch Erhitzen auf Rot- bzw. Weißglut und Abschrecken in Wasser oder speziellen Flüssigkeiten. Diese Technik wurde bei allen bislang untersuchten Saxklingen aus Oberösterreich festgestellt. Im vorliegenden Beitrag wurden erstmals zwei qualitative Gruppen herausgearbeitet. Der Vergleich der Härtewerte und Gefügebilder verdeutlicht, dass von den neun untersuchten Saxklingen vier - davon zwei aus Überackern - erfolgreich gehärtet wurden, während fünf Exemplare nur leicht erhöhte Härtewerte aufweisen.

Zusammenfassend kann festgehalten werden, dass die Saxe aus Überackern für den Kampfeinsatz geeignet waren und dem technologischen Standard ihrer Zeit entsprachen. Dabei gilt es hervorzuheben, dass zwei der Langsaxe von hoher Qualität waren.

\section{Schlüsselbegriffe}

Langsax, leichter Breitsax, Merowingerzeit, Oberösterreich, Schmiedetechnologie, Feuerverschweißen, Härtung, Metallografie.

\footnotetext{
Abstract - Typological and Technological Investigations of Seaxes from Überackern, Upper Austria

The Merovingian period cemetery of Überackern is assigned to the $7^{\text {th }}$ and early $8^{\text {th }}$ centuries AD based on documented archaeological material. The four seaxes from the cemetery were selected for typological and technological investigations as part of investigations on early medieval weapons from Upper Austria. Three of the four weapons from Überackern are long seaxes, which occur in the last third of the
}

$7^{\text {th }}$ century and represent the typical seax form of the $8^{\text {th }}$ century. The oldest exemplar belongs to the group of the light broad seaxes and dates back to the first half of the $7^{\text {th }}$ century.

The results of the metallographic analysis show that the four seaxes from Überackern have clear technological parallels. In both, the light broad seax and the three long seaxes, cutting material made of hard steel was welded to back material made of soft but more resilient iron. This method was found in seven of the nine examined seaxes from Upper Austria. Another process that can be regarded as the technological standard of the $7^{\text {th }}$ and $8^{\text {th }}$ centuries is the hardening of blades by heating them to red or white heat and quenching them in water or special liquids. This technique has been found in all seax blades from Upper Austria investigated so far. In this article, two qualitative groups were identified for the first time. A comparison of the hardness values and microstructure patterns shows that four of the nine seax blades investigated - two of them from Überackern - were successfully hardened, while five specimens had only slightly increased hardness values.

In summary, it can be said that the seaxes from Überackern were suitable for combat use and met the technological standards of their time. It is important to point out that two of the long seaxes from this cemetery were of high quality.

\section{Keywords}

Long seax, light broad seax, Merovingian period, Upper Austria, forging technology, fire welding, hardening, metallography.

\section{Einleitung}

Der vorliegende Beitrag entstand im Rahmen der Untersuchungen an frühmittelalterlichen Waffen aus Oberösterreich unter der Leitung von Erik Szameit. ${ }^{1}$ Dabei wurden die Klingenwaffen aus Bad Wimsbach-Neydharting, ${ }^{2}$ Gunskirchen-Moostal ${ }^{3}$ und Überackern sowohl typologisch als auch metallografisch untersucht. ${ }^{4}$

\footnotetext{
1 Szameit, Mehofer 2002.

2 Nau 2016, 502-504 und Taf. 10-18; 539 und Abb. 1-6; 540 und Abb. 1-6.

3 Sкомовоwsкi 2016, 549-550, 559-567 und Taf. 8-16.

4 Siehe Abschnitt 5, Technologische Untersuchungen.
} 
Vor der Abhandlung der naturwissenschaftlichen Untersuchungsergebnisse werden zunächst die verkehrsgeografisch besondere Lage des Fundorts und die typologisch-chronologische Entwicklung des Saxes eingehend beleuchtet. Die typologische Beschreibung der Saxe reicht jedoch nicht aus, um der Fülle an Informationen, die in diesen Waffen stecken, gerecht zu werden. Artefakte können allein für die Grablegung mit ausschließlich symbolischem Charakter hergestellt worden sein, oder sich tatsächlich für den Gebrauch geeignet haben. Diese Fragen sowie die technologischen Fähigkeiten der damaligen Handwerker können nur durch naturwissenschaftliche Untersuchungen gelöst werden. Ziel der metallografischen Analysen war es, den inneren Aufbau sowie die angewandten Schmiedetechnologien zu rekonstruieren und so die Grundlage für Vergleiche und weiterführende Untersuchungen zu schaffen. Die Ergebnisse dieser sowie älterer Analysen von Saxklingen aus Oberösterreich werden in diesem Beitrag gegenübergestellt und im Anschluss diskutiert. ${ }^{5}$

\section{Der Fundort Überackern}

Die vier Saxe, die für eine genauere typologische und technologische Untersuchung ausgewählt wurden, stammen aus dem merowingerzeitlichen Gräberfeld von Überackern im Westen Oberösterreichs an der Salzach-Inn-Mündung, $15 \mathrm{~km}$ südwestlich von Braunau am Inn (Abb. 1). Das heutige Dorf liegt in einer durch Schotterablagerungen der Salzach verlandeten Bucht. Überackern wurde im Jahre 768 im „Urkundenbuch des Landes ob der Enns“ zum ersten Mal schriftlich erwähnt. ${ }^{6}$

In den Jahren 1901 bis 1903 wurde das Gräberfeld von Josef Straberger, dem Vizepräsidenten des Landesmuseums Francisco Carolinum, zum Teil ausgegraben. Durch den Tod Strabergers wurden die Arbeiten am Gräberfeld mehrere Jahre eingestellt. Erst 1910 führte der ehemalige Mitarbeiter von Straberger und Mandatar des Museums, Hugo von Preen, die Ausgrabungen fort. Bis 1910 konnten 30 Gräber und ein weiteres im Jahre 1919 freigelegt werden.

\subsection{Römerzeitliche und prähistorische Strukturen}

Die merowingerzeitlichen Gräber wurden gezielt im Bereich der Ruine einer römischen Villa angelegt. Im Zuge der Ausgrabungen wurden daher mehrere römische Funde geborgen, darunter Terra Sigillata-Fragmente mit Stempeln, eine Radfibel, eine Armbrustfibel und weitere Artefakte, ${ }^{7}$

\footnotetext{
5 Siehe Abschnitt 6, Diskussion.

6 KARNITSCH 1933, 159.

7 KARNITSCH 1933, 156-162.
}

die vorwiegend in das 2. bis 3. Jh. n. Chr. datiert werden können. ${ }^{8}$ Paul Karnitsch geht davon aus, dass dieser wichtige Verkehrsknotenpunkt durch einen befestigten militärischen Posten gesichert wurde. Die als Burgstall (Kreuzlinden) bezeichnete viereckige, schanzenartige Bodenerhebung, ca. $350 \mathrm{~m}$ südöstlich des Gräberfeldes, könnte seiner Auffassung nach den Rest einer römischen Befestigungsanlage darstellen. Diese Annahme wird durch den Fund zweier römischer Münzen - ein As der jüngeren Faustina (161-164 n. Chr.) und eine Kleinbronze des Constantinus I. (324/325 n. Chr.) - sowie eines Bronzemedaillons Constantinus' I. (326 n. Chr.) aus dem Graben neben der Schanze unterstrichen. ${ }^{9}$ Bezüglich der verkehrsgeografischen Lage des Fundortes ist das urnenfelderzeitliche Gräberfeld, welches in römischer Zeit erneut für Brandbestattungen genutzt wurde, ${ }^{10}$ sowie die von Hugo von Preen vermutete einstige Römerstraße nahe des Pfarrhofes ${ }^{11}$ hervorzuheben.

\subsection{Frühmittelalterliches Gräberfeld}

Wie zuvor beschrieben, liegt das Gräberfeld an einer wichtigen antiken Verkehrsader und wurde im Bereich von römischen Gebäuderesten angelegt. Mit großer Wahrscheinlichkeit stellte der Zusammenfluss von Salzach und Inn auch im Frühmittelalter einen bedeutenden verkehrsgeografischen Knotenpunkt dar. Der Großteil der Toten wurde unter einer Wölbung aus Steinen des römischen Bauwerkes bestattet. Beispiele für die Verwendung von Spolien älterer Bauwerke finden sich häufig in Gräberfeldern der Merowingerzeit. ${ }^{12}$ Die Grablegungen in Überackern erfolgten im weichen Schwemmsand oder - im Falle von Grab 5 - auf einer Schotterschicht. Vier der insgesamt 31 Gräber, nämlich

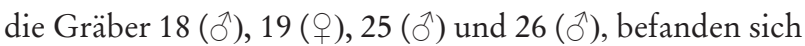
innerhalb der römischen Gebäudestruktur. ${ }^{13}$ Die Gräber 18 und 19 lagen auf dem Estrich des Erdgeschosses und hatten jeweils nur ein Eisenmesser als Beigabe. Die beigabenlosen Gräber 25 und 26 befanden sich dagegen auf dem Estrich des Hypokaustums. Die restlichen Bestattungen lagen nördlich der Gebäudestruktur und erstreckten sich bis zur Mauer des heutigen Friedhofes. Laut von Preen dürfte sich der Großteil des Gräberfeldes im Bereich des gegenwärtigen Friedhofes befinden. ${ }^{14}$

\footnotetext{
8 Für die zeitliche Zuordnung des römerzeitlichen Fundmaterials sei Julia Rabitsch gedankt.

9 Karnitsch 1933, 160-161.

10 ECKHARt 1959, 18-25.

11 KaRNITSCH 1933, 161.

12 Grunwald 2002.

13 Karnitsch 1933, 154. - Beninger, Kloiber 1962, 132.

14 KaRNITSCH 1933, 149.
} 


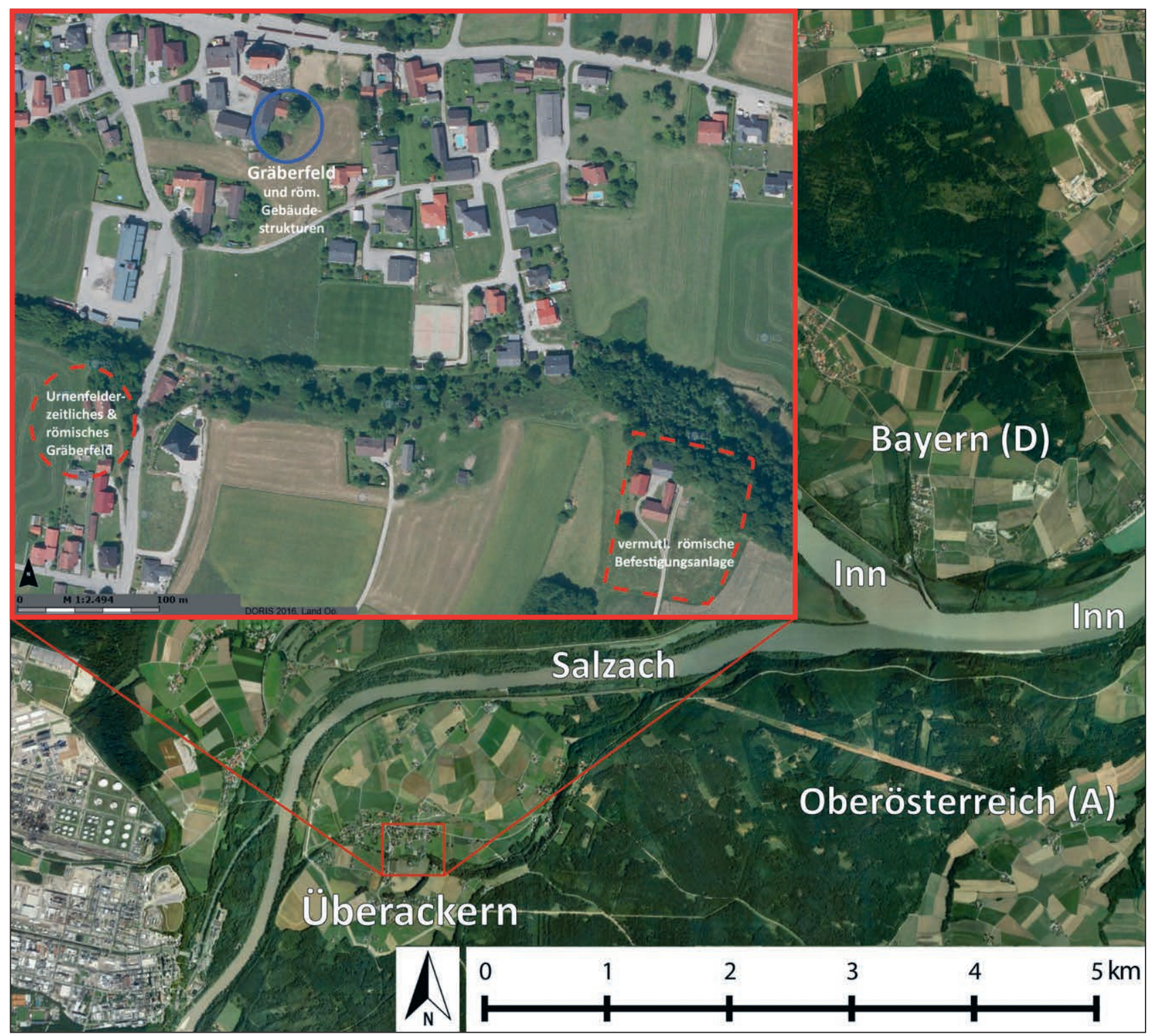

Abb. 1. Archäologische Spuren in Überackern und Kreuzlinden (Kartengrundlage: Doris - Land Oö, 2016).

Die freigelegten Gräber wurden nur teilweise beschrieben, sodass zu einigen Bestattungen keine Informationen vorliegen. Das Fundmaterial aus den Gräbern 8 bis 12 - darunter drei Saxe und Teile einer Gürtelgarnitur vom Typ Bieringen - konnte nicht mehr den einzelnen Bestattungen zugewiesen werden, da es bei einer nicht beaufsichtigten Grabung geborgen wurde. Hinsichtlich der zeitlichen Einordnung des Gräberfeldes kann gesagt werden, dass die vorliegenden Funde dem 7. und frühen 8. Jh. n. Chr. zuzuordnen sind. Anhand der Beigaben lässt sich lediglich das fundreichste Grab einem enger umrissenen Zeitrahmen zuweisen. Der im Grab 1 bestatteten Frau wurden zwei bronzene Kolbenarmringe (davon einer mit Wulstrand, der andere mit sechs Rippen, Scharnier und Verschluss), ein Ohrringpaar mit tropfenförmigen Glasanhängern und Goldfassung, zwei Riemenzungen sowie eine Gürtelschnalle beigegeben. ${ }^{15}$ Das Grabinventar kann in die Zeit des Übergangs vom 7. zum 8. Jh. datiert werden. ${ }^{16}$ Anhand der Saxe kann ebenfalls ein Schwerpunkt in dieser Zeit

15 Karnitsch 1933, 149-150 und Abb. 68. - Beninger, Kloiber 1962, Taf. 5-7.

16 WüHRER 2000, 46, 185 ordnet die Kolbenarmringe der Schicht 4 nach Koch zu, welche der Übergangsphase vom 7. zum 8. Jh. entspricht. - Für diese Phase sind auch die Ohrringe mit tropfenförmigen Glasanhängern belegt: Косн 1982, 50. 
festgestellt werden. Bei drei der vier Waffen handelt es sich um Langsaxe, die im letzten Drittel des 7. Jhs. auftreten und die typische Saxform des 8. Jhs. darstellen. ${ }^{17}$ Das älteste Exemplar zählt zur Gruppe der leichten Breitsaxe, die in die Zeit zwischen dem späten 6. Jh. und die Mitte des 7. Jhs. datieren. Da aus den freigelegten Gräbern keine Funde des 6. Jhs. vorliegen, datiert der leichte Breitsax aus Überackern mit großer Wahrscheinlichkeit in die erste Hälfte des 7. Jhs.

\section{Der Sax: Das einschneidige Kurzschwert des Frühmittel-} alters

Der Sax ist die am häufigsten beigegebene Waffe in den merowingerzeitlichen Reihengräbern östlich des Rheins. ${ }^{18} \mathrm{Die}$ Bezeichnung Sax wird erstmals von Gregor von Tours im späten 6. Jh. verwendet. Er bezeichnete die Waffe als Scramasax und beschrieb sie als schweres Messer mit vergifteter Klinge. ${ }^{19}$ Im Vergleich mit der zweischneidigen Spatha zeigt die Saxklinge einen keilförmigen Querschnitt, weshalb sie wesentlich starrer als die Spatha ist. ${ }^{20}$ Die beiden Waffen unterscheiden sich also durch ihre Form und Länge, aber auch durch ihre Trageweise. Die Spatha wurde nur von einer ausgewählten, kleinen und sozial hochgestellten Gruppe an einem speziellen Wehrgehänge getragen. Der Sax hingegen wurde vom Großteil der Männer unterschiedlicher gesellschaftlicher Schichten getragen. Die Waffe steckte in einer Scheide, die am Leibgurt (Hauptgürtel), der das Gewand zusammenhielt, befestigt war. ${ }^{21}$ Die Scheiden selbst waren aus Leder und/oder Holz, jene aus Leder waren an der Unterseite vernietet. Ihre Reste finden sich an der Schneidenseite der Waffe in Form von Nietenreihen aus Bronze, mitunter auch größeren Ziernieten oder Zierplatten, wie z. B. die Saxscheidenverzierung aus Hörpolding (OÖ). ${ }^{22}$

Hinsichtlich der Frage nach der Symbolik gilt es zu betonen, dass die frühen Saxe meist Spezialanfertigungen höhergestellter Persönlichkeiten waren. Ab dem 6. Jh. wurde die Waffe Männern bis ins hohe Alter sowie Knaben verschiedener sozialer Gruppen mit ins Jenseits gegeben. So findet man den Sax neben Lanze, Schild und Spatha in

17 Szameit 1987, 162. - Szameit, Mehofer 2002, 130. - Siehe hierzu auch Koch 1994.

18 Siegmund 1996, 705.

19 Gregor von Tours, Libri historiarum decem IV,51: „Tunc duo pueri cum cultris validis, quos vulgo scramasaxos vocant, infectis vinino, malificati a Fredegundae regina, cum aliam causam suggerire simularent, utraque ei latera feriunt".

20 WERnARD 1998, 749.

21 Siegmund 1996, 701

22 ZELLER 1988, 240. reichen Kriegerbestattungen sowie als oft einzige Waffe in einfach ausgestatteten Gräbern. Da der Sax am Leibgurt getragen wurde, dürfte er einen fixen Trachtbestandteil des Mannes dargestellt haben. Vermutlich hatte die Waffe einen symbolisch-repräsentativen Charakter. Naheliegend erscheint, dass dadurch die Zugehörigkeit zu einer gewissen gesellschaftlichen Gruppe gezeigt wurde. Die große Zahl der Saxe mit vergleichbaren Dimensionen und technologischen Merkmalen legt die Vermutung nahe, dass sie in regionalen Fabrikationszentren produziert wurden. ${ }^{23}$ Bislang konnte diese Hypothese allerdings nicht bestätigt werden.

\subsection{Geschichte der Saxforschung}

In den 80er Jahren des 19. Jhs. unterteilte Ludwig Lindenschmit die Waffengattung des Saxes anhand der Maßunterschiede in typologische Gruppen. ${ }^{24}$ Er vermutete, dass die unterschiedlichen Saxtypen aus derselben Zeit stammten und ihre uneinheitlichen Dimensionen auf verschiedene Verwendungszwecke zurückzuführen seien. Lindenschmit unterstrich seine Typologie durch die Benennungen Sax, Scramasax und Langsax aus historischen Quellen, denen er die verschiedenen Gruppen zuwies. Jedoch erwähnen diese schriftlichen Überlieferungen nichts über die Dimensionen und Verwendung der Waffe. ${ }^{25}$

Eduard Brenner widersprach der von Lindenschmit aufgestellten Hypothese, da er davon ausging, dass die unterschiedlichen Dimensionen verschiedene Entwicklungsstufen dieser Waffenform darstellen. ${ }^{26}$ Seiner Auffassung, die Form und Dimension eines Saxes stelle ein chronologisches Element dar, schlossen sich schließlich andere Wissenschaftler im Groben an.

Anhand der typologischen Merkmale unterschied Kurt Böhner zwischen den drei Gruppen der Schmal-, Breit- und Langsaxe. ${ }^{27}$ Diese Dreiteilung reichte nicht aus, um der Variationsbreite dieses Waffentyps gerecht zu werden, weshalb Christiane Neuffer-Müller für das Gräberfeld SontheimBrenz zusätzlich die Gruppe der Kurzsaxe definierte.28 Umfangreiche Studien zur Typologie und Chronologie des einschneidigen Schwertes im Frühmittelalter wurden schließlich von Jo Wernard für den süddeutschen Raum, von Frank Siegmund für den Niederrhein und von Herbert Westphal für das sächsische Stammesgebiet durchgeführt. ${ }^{29}$

\footnotetext{
23 WERNARD 1998, 776.

24 LindenSCHMIT 1880-1889, 204.

25 BÖHNER 1958, 130.

26 BRENNER 1912, 290.

27 BÖHNER 1958, 130-145.

28 NeufFer-Müller 1966, 28.

29 Westphal 1991. - Siegmund 1998. - Wernard 1998.
} 
Zuletzt wurden Arbeiten zu den awarenzeitlichen Saxen aus dem Karpatenbecken ${ }^{30}$ und aus Slowenien ${ }^{31}$ vorgelegt.

Wernard weist darauf hin, dass sich in der Literatur die Ergebnisse der verschiedenen Archäologen stets unterscheiden. Dies führt er darauf zurück, dass die Untersuchungen meist im Rahmen von Gräberfeldanalysen mit begrenzter Fundmenge erfolgten. Um eine einheitlichere Typologie und Chronologie zu erarbeiten, verglich er insgesamt 785 Saxe aus ganz Süddeutschland. Er orientierte sich an der von Rainer Christlein erarbeiteten Stufengliederung des Gräberfeldes von Marktoberdorf, ${ }^{32}$ welche jedoch von aktuellen Chronologiesystemen ${ }^{33}$ abweicht. Siegmund versuchte anhand von 137 Saxen eine Gliederung dieser Waffe für den Niederrhein zu erarbeiten. ${ }^{34}$ Er sieht keine Notwendigkeit einer eigenen Gruppe der Kurzsaxe und zählt diese zu den Schmalsaxen bzw. leichten Breitsaxen. Basierend auf Siegmunds Vorarbeiten erweiterte die Arbeitsgruppe Franken AG die Materialbasis um 142 Exemplare und kam dabei zu denselben typologischen sowie chronologischen Ergebnissen. ${ }^{35}$

Westphal untersuchte 114 Saxklingen aus dem sächsischen Stammesgebiet in Nordwestdeutschland auf die angewandte Schmiedetechnik, ihre Typologie und Dekoration. ${ }^{36}$ Die typologischen Vergleiche zeigten, dass die Klingen aus diesem Gebiet deutliche Unterschiede zu jenen aus dem süddeutschen Raum aufweisen und durch eine Reihe von Eigenarten gekennzeichnet sind. Westphal betont, dass sächsische Saxe daher nur in Einzelfällen mit jenen aus dem fränkischen und alamannischen Stammesgebiet verglichen bzw. den von Böhner ${ }^{37}$ und Koch ${ }^{38}$ entwickelten Kriterien zugeordnet werden können. ${ }^{39}$

Die typologischen Unterschiede, besonders zwischen dem süd- und norddeutschen Raum verdeutlichen, dass es zu regionalen Entwicklungen gekommen ist. In Skandinavien, ${ }^{40}$ Norddeutschland ${ }^{41}$ und England ${ }^{42}$ bildeten sich teils andere Formen heraus und gewisse Saxtypen treten erst später als in Mitteleuropa auf. Im vorliegenden Beitrag wird daher die von Wernard für den süddeutschen Raum erarbeitete

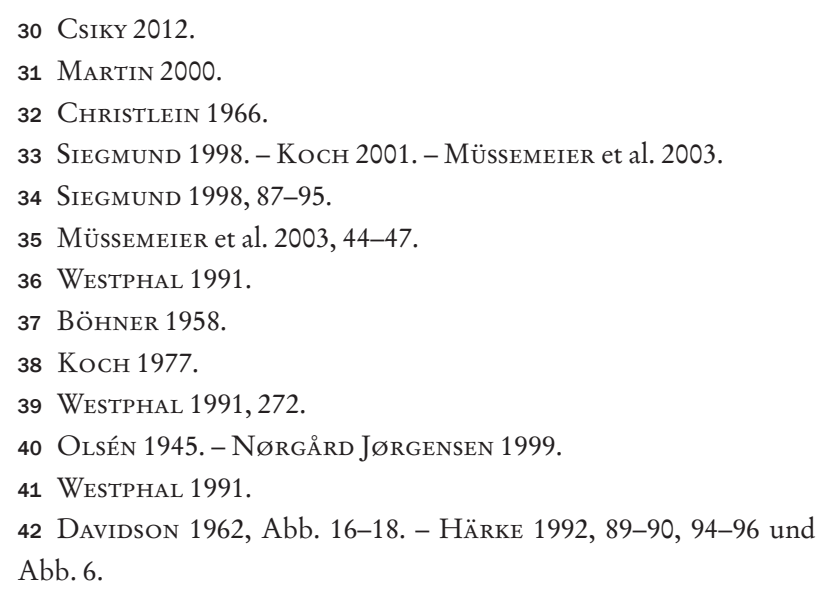

typologische Gliederung unter Berücksichtigung aktueller Chronologiesysteme ${ }^{43}$ angewandt.

\subsection{Chronologisch-typologische Entwicklung des Saxes in Mitteleuropa}

Die typologischen Merkmale eines Saxes sind die Klingenlänge und -breite, die Länge der Griffangel, die Klingenform sowie eventuelle Verzierungen. Für die chronologische Gliederung sind neben den Maßen und formalen Merkmalen auch die Beifunde zu berücksichtigen. Da der Sax am Leibgurt getragen wurde, können bestimmte Gürtelformen mit definierten Saxtypen in Korrelation gebracht werden. ${ }^{44}$ Trotz der zuvor erwähnten unterschiedlichen Gliederungen lässt sich die Saxentwicklung anhand der Typologie Wernards und unter Berücksichtigung aktueller Chronologiesysteme ${ }^{45}$ für den alamannisch-bajuwarischen Raum wie folgt zusammenfassen: ${ }^{46}$

\subsubsection{Schmalsax}

Die früheste Saxform ist der lange Schmalsax der zweiten Hälfte des 5. Jhs., der im Gegensatz zu späteren Formen innerhalb eines eng begrenzten Zeitraumes, dem sogenannten Goldgriffspatha-Horizont, in Erscheinung tritt. ${ }^{47}$ Dieser Typ besitzt eine schlanke, lange Klinge mit Klingenlängen von ca. 33-46 cm und -breiten von ca. 2,6-3,2 cm. Da diese seltenen Formen meist prunkvolle Unikate sind, die wohl Spezialanfertigungen für höherrangige Personen waren, weist der lange Schmalsax eine große Variationsbreite an Proportionen auf. ${ }^{48}$ Vergleiche finden sich besonders in Osteuropa ${ }^{49}$ weshalb der Westwanderung sarmatisch-alanischer Gruppen eine nicht zu unterschätzende Rolle für das Auftreten dieser Waffe in Mittel- und Westeuropa zuzusprechen ist.

\subsubsection{Kurzsax}

Im letzten Viertel des 5. Jhs. geht der Trend hin zu Saxen mit kürzeren Klingen, welche die charakteristischen Formen des 6. Jhs. darstellen. Die Klingen dieser Typen sind zwischen

\footnotetext{
43 Siegmund 1998. - Koch 2001. - Müssemeier et al. 2003.

44 WERNARD 1998, 748.

45 Siegmund 1998. - Koch 2001. - Müssemeier et al. 2003.

46 An dieser Stelle gilt es darauf hinzuweisen, dass die Minimal- und Maximalwerte der Längenmaße ein bis zwei Zentimeter und die der Breitenmaße ein bis zwei Millimeter variieren können. Aus diesem Grund wurden die Maße, bei denen der Wert etwas kleiner oder größer sein kann, mit „ca.“ angegeben.

47 WERNARD 1998, 772.

48 WeRnARD 1998, 772.

49 Косн 2001, 279, 281 und Abb. 113; 573-575 und Liste 31. - Kiss 2014.
} 
ca. 2,5-3,5 cm breit, wobei Wernard jene Exemplare mit Klingenlängen von ca. $18-30 \mathrm{~cm}$ als Kurzsaxe, die längeren Varianten mit ca. 30-36 cm langen Klingen als Schmalsaxe bezeichnet. ${ }^{50}$ Mit den beschriebenen Maßen ist der Sax des 6. Jhs. oft nur wenig länger als ein einfaches Messer. Durch die kürzeren Klingen zeichnet sich eine Veränderung in der Funktion der Waffe ab und wirft die Frage auf, inwieweit diese Typen als Weiterentwicklung des langen Schmalsaxes anzusehen sind. Während der Großteil der Forscher einen klaren Bezug zu den langen Formen des 5. Jhs. sieht, vermuten einige einen Zusammenhang mit den germanischen Kampfmessern Nordeuropas, ${ }^{51}$ wieder andere mit den Waffen des spätrömischen Heeres. ${ }^{52}$

In der zweiten Hälfte des 6. Jhs. begann sich eine Entwicklung zu schwereren, breiteren Klingen abzuzeichnen. In dieser Zeit traten neben den leichten Saxtypen erstmals der massive Kurzsax sowie der leichte Breitsax in Erscheinung. Der massive Kurzsax ist eine breitere Ausformung des Kurzsaxes und weist bei einer Klingenlänge zwischen ca. $22 \mathrm{~cm}$ und $30 \mathrm{~cm}$ eine Klingenbreite von $3,5-4 \mathrm{~cm}$, in manchen Fällen bis zu $5 \mathrm{~cm}$ auf. ${ }^{53}$

\subsubsection{Leichter Breitsax}

Der leichte Breitsax entwickelte sich im späten 6. Jh. und war bis zur Mitte des 7. Jhs. in Verwendung. Diese Saxform hebt sich mit einer Klingenlänge von ca. 26-42 cm und -breite von 4-5 cm sowie einer längeren Griffangel von den Kurzsaxformen ab. ${ }^{54}$ Während die Griffangeln der früheren Formen die Länge von $20 \mathrm{~cm}$ nicht übersteigen, weisen jene mancher Stücke der ersten Hälfte des 7. Jhs. Längen von über $20 \mathrm{~cm}$ auf. ${ }^{55}$ Die Länge der Handhaben der leichten Breitsaxe variiert noch im 7. Jh. und kann daher nur bedingt als chronologisches Element angesehen werden. Die Herausbildung des leichten Breitsaxes stellt den Beginn der Entwicklung des Saxes von einer Stichwaffe zu einer Hiebwaffe dar. Durch die breiteren und längeren Klingen sowie die längeren Griffangeln eignete sich diese Saxform deutlich besser für den Hieb. Dieser Entwicklungsprozess führte dazu, dass sich während der ersten Hälfte des 7. Jhs. der schwere Breitsax herausbildete und der Kurzsax aufgegeben wurde. Durch das Verschwinden der Kurzsaxe und

50 Wernard 1998, 771 und Abb. 3.

51 LOSERT 2003, 412.

52 Martin 1993. - Martin 2000.

53 Wernard 1998, 771 und Abb. 3; 775.

54 Nicht selten finden sich Exemplare, die zu schmal für die Gruppe der Breitsaxe und zu lang für jene der Kurzsaxe ist. Wernard 1998, 771 und Abb. 3 bezeichnet diese Variante als Leichtsax.

55 WERNARD 1998, 776. der zunehmenden Dominanz der schweren Breitsaxe ist der Sax spätestens um die Mitte des 7. Jhs. nicht mehr als reine Stich- sondern auch als Hiebwaffe anzusehen. Obwohl die späten Typen - der Breitsax und der Langsax - Hiebwaffen darstellen, erlaubte es die spitze Klingenform, sie dennoch für den Stich einzusetzen.

\subsubsection{Schwerer Breitsax}

Der schwere Breitsax weist Klingenlängen von ca. 34-46 cm und -breiten von über $5 \mathrm{~cm}$ auf. Er besitzt fast durchwegs sehr lange Griffangeln mit über $25 \mathrm{~cm}$ Länge. Dieser Saxtypus kommt neben leichten Breitsaxen in der ersten Hälfte und gemeinsam mit Langsaxen in der zweiten Hälfte des 7. Jhs. vor. ${ }^{56}$ Die sehr lange, im Verhältnis zur Klinge überdimensional wirkende Griffangel ermöglichte es, die Waffe unterschiedlich einzusetzen. Angesichts des Aufbaus dieser Saxform erscheint eine zweihändige Führung nur wenig effektiv. Für den beidhändigen Einsatz müsste die Klinge deutlich länger sein und der Schwerpunkt im vorderen Viertel liegen, um die Hiebkraft zu verstärken. Folglich ist der schwere Breitsax einhändig geführt worden und der lange Griff diente dazu, die Waffe - je nach Bedarf - einzusetzen. Wurde der Sax im hinteren Bereich des Griffs gehalten, konnte die volle Hiebkraft entfaltet werden. Hielt man die Waffe im vorderen oder mittleren Bereich des Griffes, konnte sie besser zum Stechen eingesetzt werden. Dieser Überlegung zufolge handelt es sich beim schweren Breitsax um eine Weiterentwicklung der massiven, schweren Saxformen, die für den Kampf zu Fuß konzipiert war. Die einhändige Waffenführung ermöglichte es zudem einen Schild zu tragen.

\subsubsection{Langsax}

Im letzten Drittel des 7. Jhs. tritt erstmals der Langsax in Erscheinung, der zur charakteristischen Form der späten Merowinger- und frühen Karolingerzeit wird. ${ }^{57}$ Wolfgang Hübener sieht in den längeren und schlanken Breitsaxformen eine Übergangsform zum Langsax und nennt sie „langsaxähnlich“. ${ }^{58}$ Der Langsax besitzt Klingen mit Längen von ca. $48 \mathrm{~cm}$ bis über $65 \mathrm{~cm}$ und Breiten von ca. 3,8$4,8 \mathrm{~cm}$. Die Griffangeln werden bei dieser Saxform wieder kürzer, was auf eine großteils einhändige Waffenführung schließen lässt. Dennoch liegen mehrere Exemplare - wie der Langsax A-1784 aus Überackern - vor, bei denen die Länge der Angel - in diesem Falle 18,5 cm - eine beidhändige Waffenführung erlaubt. Mit den beschriebenen

56 Siegmund 1998, 94. - Müssemeier et al. 2003, 46.

57 Szameit 1987, 162, 70, 130. - Косн 1994.

58 HÜBENER 1989. 
Klingenlängen ist der Langsax meist nur wenig kürzer als die zweischneidige Spatha und verliert dadurch den Charakter eines Kurzschwertes. Der Langsax bleibt die dominierende Saxform des 8. Jhs., wobei durch das Ausdünnen der Beigabensitte immer weniger Stücke als Grabfunde überliefert sind. Aufgrund der großen Unterschiede zu den Vorgängerformen sehen manche Forscher den Langsax als neuen Waffentyp an, der sowohl technologisch als auch funktional anders konzipiert ist. ${ }^{59}$ Derartige Überlegungen sind vergleichbar mit der zuvor thematisierten Frage, ob der Kurzsax tatsächlich eine Weiterentwicklung des langen Schmalsaxes darstellt. Dabei sei darauf hingewiesen, dass beide Entwicklungssprünge die Dimensionen der Waffe betrafen, während die jeweilige Grundfunktion - Stich- oder Hiebwaffe - sowie die Trageweise am Leibgurt zunächst dieselbe blieb. Erst die großen Langsaxe des frühen 8. Jhs. wurden teilweise an einem eigenen Wehrgehänge getragen, wie dies beispielsweise für den Langsax mit prunkvoller Scheide aus Dürbheim (Baden-Württemberg) diskutiert wird..$^{60}$

\subsubsection{Klingenform}

Neben den Dimensionen stellt die Klingenform ein datierendes Element dar, das in drei Typen (Abb. 2) unterteilt und für den süddeutschen-österreichischen Raum wie folgt gegliedert werden kann: ${ }^{61}$

- Typ 1 = die Schneide zieht zum Rücken hinauf, sodass die Spitze auf der Höhe des Rückens liegt,

- Typ 2 = der Rücken fällt mehr oder weniger steil zur Schneide hin ab, sodass die Spitze etwa auf der Höhe der Schneide liegt,

- Typ 3 = der Rücken und die Schneide laufen etwa parallel, sodass die Spitze ungefähr auf der Mittelachse der Klinge liegt.

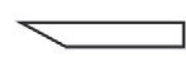

Typ 1

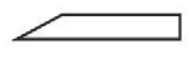

Typ 2

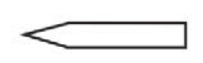

Typ 3
Abb. 2. Klingenformen (nach Wernard 1998, Tab. 1).

Typ 1 ist bei den frühen Formen, dem langen Schmalsax der Zeit vor 500 und auch dem Schmal- und Kurzsax des 6. Jhs. am häufigsten nachweisbar. In der zweiten Hälfte des

\footnotetext{
59 WestPhal 1991.

60 Krohn 2013, 356 und Abb. 1.

61 WERNARD 1998, 749.
}

6. Jhs. kommen größere und massivere Saxtypen in Mode, mit denen sich ein Wandel zu Typ 3 abzeichnet, welcher dann bei den Breitsaxen des 7. Jhs. dominiert. In der späten Merowingerzeit, vom ausgehenden 7. Jh. bis in die Mitte des 8. Jhs., treten vor allem im bajuwarischen Raum Saxklingen vom Typ 2 auf. $^{62}$

\section{Typologie und Chronologie der Saxe aus Überackern}

Im Folgenden werden die vier Saxe (Taf. 1) einzeln beschrieben, typologisch eingeordnet und datiert. Eingangs werden Informationen über die Fundzusammenhänge gegeben.

\section{1. Überackern, Grab 3: Langsax (A-1775)}

In diesem Grab wurde ein ca. 50-jähriger Mann bestattet. Außer dem Langsax wurden keine weiteren Beigaben dokumentiert.

\subsubsection{Beschreibung und Datierung des Langsaxes A-1775}

$\begin{array}{ll}\text { Gesamtlänge: } & 63 \mathrm{~cm} \\ \text { Griffangellänge: } & 14 \mathrm{~cm} \\ \text { Klingenlänge: } & 49 \mathrm{~cm} \\ \text { Klingenbreite: } & 4,6 \mathrm{~cm}\end{array}$

Der Sax ist über die gesamte Länge erhalten und ist von einer stärkeren Korrosionsschicht überzogen. Lediglich in wenigen Bereichen ist die Schneide ausgebrochen. Der Rücken und die Schneide sind gerade und verlaufen parallel zueinander. Im vorderen Bereich laufen die Klingen und der Rücken aufeinander zu, sodass die Spitze ungefähr auf der Mittelachse, jedoch etwas näher an der Schneide liegt. Damit entspricht die Klingenform dem Typ 3 mit einer leichten Tendenz zu Typ 2. Die Griffangel ist von der Schneide abgesetzt. Vom Rücken zieht die Angel leicht nach unten, sodass der Griff ungefähr auf Höhe der Mittelachse der Klinge liegt. Unterhalb des Rückens verläuft eine gerade Blutrille, die aufgrund der Korrosion nur mehr ansatzweise zu erkennen ist. Aufgrund der Proportionen und Klingenform - Typ 3 mit Tendenz zu Typ 2 - dürfte es sich um einen Langsax des frühen 8 . Jhs. handeln.

\section{2. Überackern, Gräber 8-12: leichter Breitsax (A-1783) und Langsaxe (A-1776 und A-1784)}

Beim Ausheben der Gräber 8-12 wurde leider nicht auf die Fundzusammengehörigkeit geachtet, sodass man die Funde heute keinem Grab mehr zuordnen kann. Aus den fünf Gräbern kennen wir drei Saxe, dabei handelt es sich um einen leichten Breitsax und zwei Langsaxe. Daneben

62 WeRNARD 1998, 750 


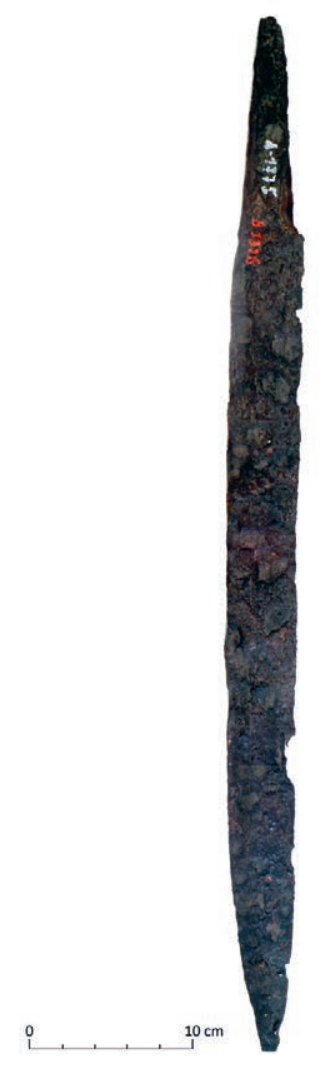

Langsax A-1775
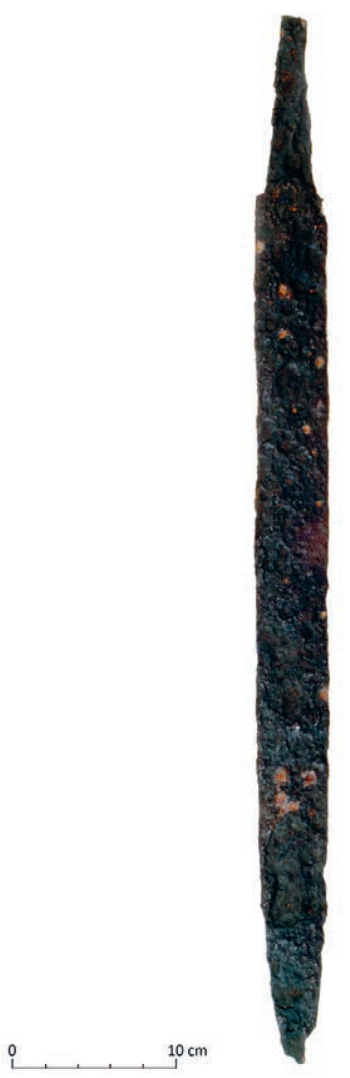

Langsax A-1776
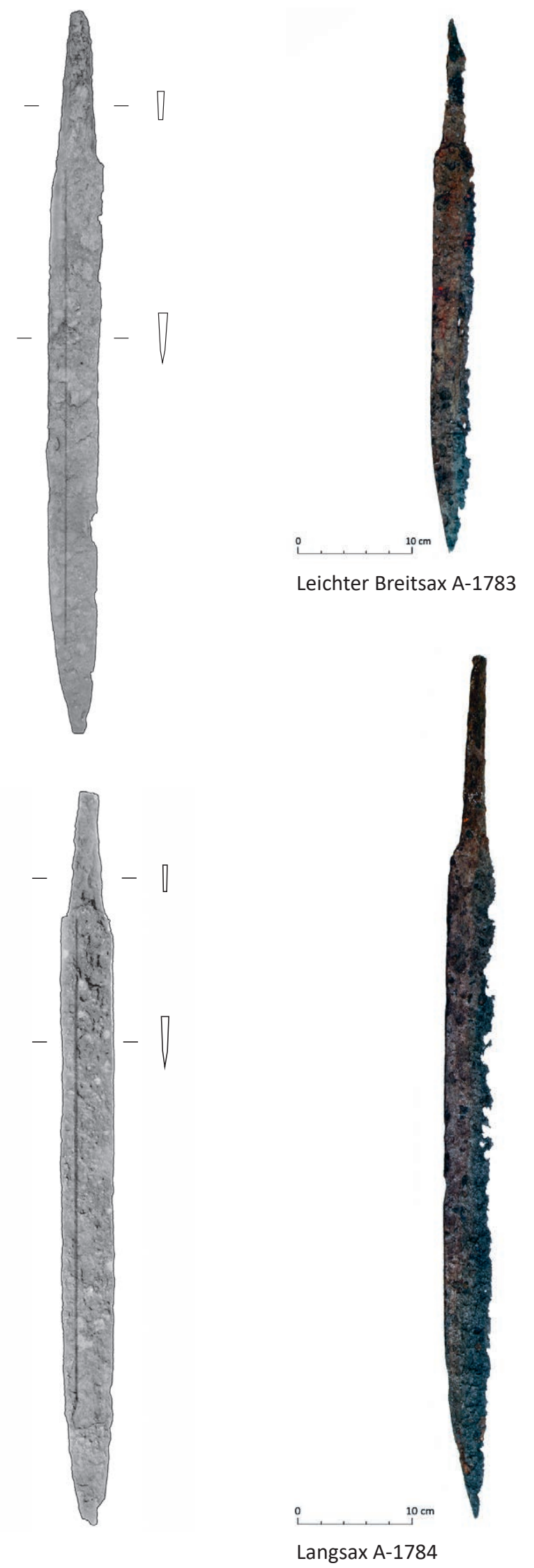
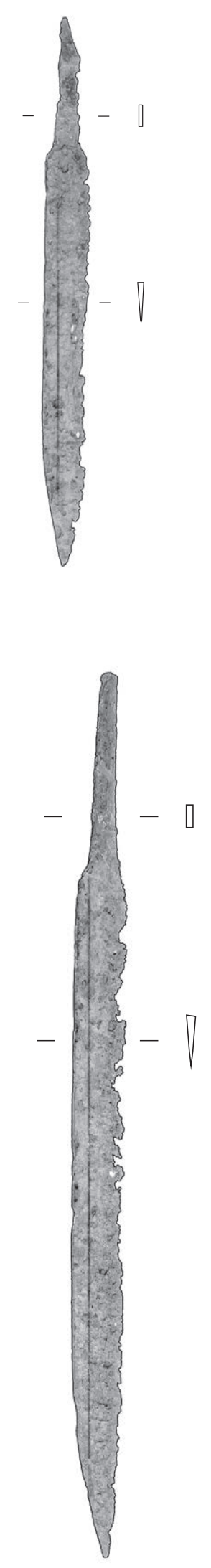

Taf. 1. Die untersuchten Saxe aus dem Gräberfeld von Überackern. 
stammen drei Griffangelmesser, von denen nur mehr eines vorhanden ist (Inv. Nr. A-1771), aus diesen fünf Gräbern. Es hat eine Länge von $10,3 \mathrm{~cm}$, wobei die Griffangel zum größten Teil abgebrochen ist. Weitere Funde aus den Gräbern 8-12 sind zwei Teile einer vielteiligen Gürtelgarnitur vom Typ Bieringen, eine bronzene Schilddornschnalle (Inv. Nr. A-1796) sowie eine $8 \mathrm{~cm}$ lange und 2,3 cm breite Riemenzunge aus Bronze mit drei Nieten (Inv. Nr. A-1791). Gürtelgarnituren des Typs Bieringen stellen Leibgurte dar, welche ab der ersten Hälfte des 7. Jhs. vorkommen ${ }^{63}$ und häufig in Kombination mit einem Sax gefunden wurden. Es ist daher naheliegend, dass der Gürtel gemeinsam mit einem der drei Saxe ins Grab gelangte. Da die ersten dieser Gürtel bereits vor der Mitte des 7. Jhs. im Umlauf waren, kann nicht ausgeschlossen werden, dass die Garnitur mit dem leichten Breitsax in Verbindung steht. Das vermehrte Vorkommen der Gürtelgarnituren vom Typ Bieringen in Gräbern der zweiten Hälfte des 7. Jhs. lässt jedoch eher auf den Zusammenhang mit einem der Langsaxe schließen.

\subsubsection{Beschreibung und Datierung des Langsaxes A-1776}

$\begin{array}{ll}\text { Gesamtlänge: } & 66 \mathrm{~cm} \\ \text { Griffangellänge: } & 10,5 \mathrm{~cm} \\ \text { Klingenlänge: } & 55,5 \mathrm{~cm} \\ \text { Klingenbreite: } & 4,5 \mathrm{~cm}\end{array}$

Der Sax ist über die gesamte Länge erhalten und von einer stärkeren Korrosionsschicht überzogen. Die Schneide ist bis auf wenige Stellen gut erhalten. Der Rücken und die Schneide sind gerade und verlaufen parallel zueinander. Im vorderen Bereich laufen die Klingen und der Rücken aufeinander zu, sodass die Spitze ungefähr auf der Mittelachse liegt. Die Klingenform entspricht somit dem Typ 3. Die Griffangel ist von der Schneide und vom Rücken abgesetzt. Der Griff liegt ungefähr auf Höhe der Mittelachse der Klinge. Unterhalb des Rückens verläuft eine gerade Blutrille, die aufgrund der Korrosion nur mehr ansatzweise zu erkennen ist. Proportionen und Klingenform dieses Langsaxes sprechen für eine Datierung in das ausgehende 7. Jh. oder frühe 8. Jh.

Langsaxe mit der Klingenform vom Typ 3 finden sich im Kriegergrab aus Enns-Lauriacum, ${ }^{64}$ in Rudelsdorf bei Linz (Gräber 7, 8, 13 und 38) ${ }^{65}$ und in Bad Wimsbach-Neydharting (Grab 9, 10 und 56). ${ }^{66}$ Die Exemplare aus Rudelsdorf sowie Bad Wimsbach werden in die Zeit um oder kurz nach 700, das Kriegergrab aus Enns in das frühe 8. Jh. datiert.

\footnotetext{
63 Freundlicher Hinweis Bendeguz Tobias.

64 Szameit, Mehofer 2002, 127-129 und Abb. 1; 138 und Abb. 6/C.

65 Csar 2002, 248-251 und Taf. 2/1, 2/3, 4/2, 7/3.

66 Nau 2016, 502 und Taf. 4/A, 7/B, 10.
}

\subsubsection{Beschreibung und Datierung des leichten Breitsaxes} A-1783

$\begin{array}{ll}\text { Gesamtlänge: } & 47 \mathrm{~cm} \\ \text { Griffangellänge: } & 11 \mathrm{~cm} \\ \text { Klingenlänge: } & 36 \mathrm{~cm} \\ \text { Klingenbreite: } & 4 \mathrm{~cm}\end{array}$

Der Sax ist großteils erhalten, wobei das Ende der Griffangel gebrochen sein könnte. Durch Korrosion sind über die gesamte Länge der Schneide kleine Bereiche ausgerissen und einige Löcher entstanden. Der Rücken und die Schneide sind gerade und verlaufen parallel zueinander. Im vorderen Bereich laufen die Klinge und der Rücken aufeinander zu, sodass die Spitze ungefähr auf der Mittelachse liegt. Damit entspricht die Klingenform dem Typ 3. Die Griffangel ist von der Schneide und vom Rücken abgesetzt, sodass der Griff ungefähr auf Höhe der Mittelachse der Klinge liegt. Unterhalb des Rückens verläuft eine gerade Blutrille, die nur mehr leicht zu erkennen ist. Aufgrund der Proportionen und der Klingenform könnte dieses Exemplar sowohl in das späte 6. Jh. als auch in die erste Hälfte des 7. Jhs. datieren. Da aus Überackern keine Funde aus der Zeit vor 600 n. Chr. bekannt sind, erscheint eine Datierung in die erste Hälfte des 7. Jhs. plausibel.

Als Vergleichsexemplar kann der leichte Breitsax aus Gunskirchen-Moostal herangezogen werden, für den ebenfalls eine Datierung in die erste Hälfte des 7. Jhs. angenommen wird. ${ }^{67}$

\subsubsection{Beschreibung und Datierung des Langsaxes A-1784}

$\begin{array}{ll}\text { Gesamtlänge: } & 78 \mathrm{~cm} \\ \text { Griffangellänge: } & 18,5 \mathrm{~cm} \\ \text { Klingenlänge: } & 59,5 \mathrm{~cm} \\ \text { Klingenbreite: } & 4,3 \mathrm{~cm}\end{array}$

Der Sax ist nahezu gänzlich erhalten, wobei durch Korrosion über die gesamte Länge der Schneide kleine Bereiche ausgerissen und einige Löcher im Schneidenbereich entstanden sind. Der Rücken und die Schneide sind gerade und verlaufen über drei Viertel der Klingenlänge parallel zueinander. Im vorderen Viertel zieht der Rücken zur Schneide hinunter. Die Spitze kommt dabei unterhalb der Mittelachse zu liegen, sodass die Klinge dem Typ 2 zuzuordnen ist. Die Griffangel ist vom Rücken abgesetzt, während der Übergang zur Schneide nicht mehr eindeutig zu erkennen

67 SкомоRowski 2016, 547-548 und Abb. 8. 


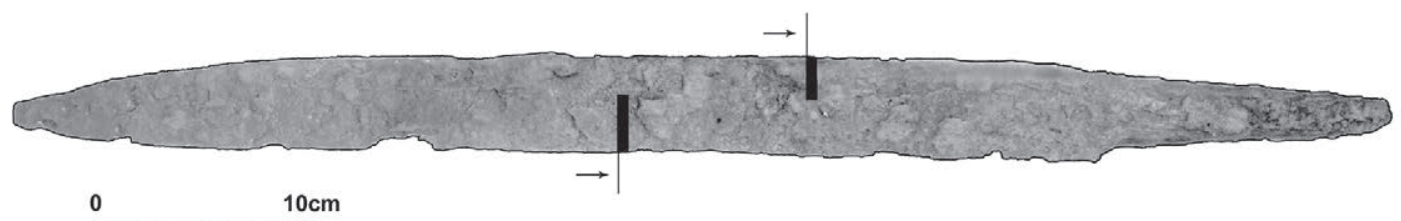

Abb. 3. Probenmaterial vom Langsax A-1775.

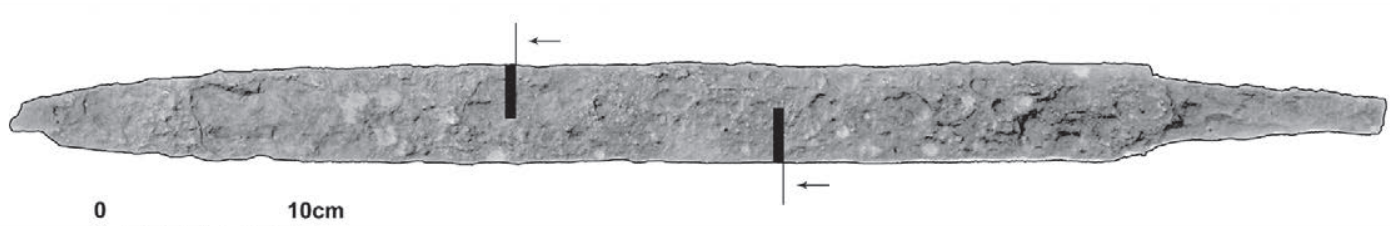

Abb. 4. Probenmaterial vom Langsax A-1776.

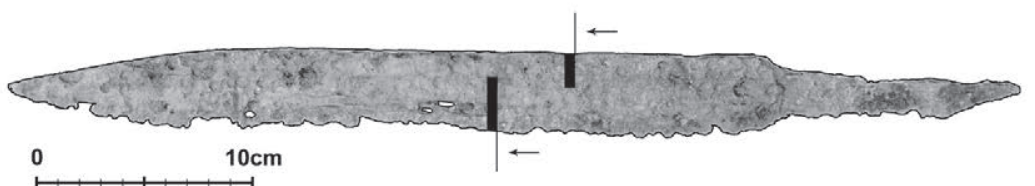

Abb. 5. Probenmaterial vom leichten Breitsax A-1783.

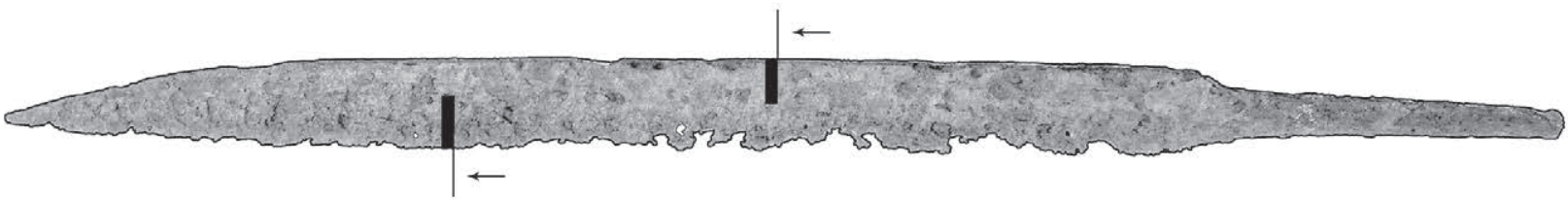

0

$10 \mathrm{~cm}$

Abb. 6. Probenmaterial vom Langsax A-1784.

ist. Die Griffangel liegt leicht unterhalb der Mittelachse der Klinge. Die Klingenform vom Typ 2 spricht dafür, dass es sich um eine fortgeschrittene Langsaxform der ersten Hälfte des 8. Jhs. handelt.

\section{Technologische Untersuchungen}

Die metallografische Analyse der beschriebenen Waffen soll Einblick in die Schmiedetechnologie der Merowingerzeit in Oberösterreich bzw. dem östlichen Herrschaftsgebiet der Bajuwaren gewähren. Erste Untersuchungen zur Technologie frühmittelalterlicher Waffen aus Österreich wurden von Erik Szameit bereits in den 1980er Jahren vorgenommen, wobei sich diese auf röntgentechnologische Analysen beschränkten. ${ }^{68}$ Diese Methode erlaubt es, den Aufbau einer Klinge zu erkennen, gibt jedoch nur bedingt Auskunft über die Qualität des Materials sowie der Schmiede- und Härtungstechnologie. Um diese Eigenschaften der früh- und hochmittelalterlichen Waffen festzustellen, veranlasste Szameit schließlich systematische metallografische Analysen, welche durch den Archäometallurgen Mathias Mehofer durchgeführt wurden. ${ }^{69}$ Es handelt sich dabei um

68 Szameit 1986, 385.

69 Szameit, Mehofer 2002. 
die ersten technologischen Untersuchungen an frühmittelalterlichen Waffen für diese Region. Die vorliegenden technologischen Untersuchungsergebnisse wurden im Rahmen dieser Forschungen im metallografischen Labor des Vienna Insitute for Archaeological Science (VIAS) erarbeitet.

Ziel der Untersuchung war es, die Schmiedetechnologie und die Qualität der Waffen zu beurteilen. In weiterer Folge können die Ergebnisse mit jenen anderer Saxe verglichen werden, um technologische Gemeinsamkeiten sowie Unterschiede festzustellen. ${ }^{70}$ Die Vergleiche sollen dazu beitragen, eventuelle Werkstättenkreise sowie technologische Traditionen und Innovation nachzuweisen. Vorbild der Studien sind die umfangreichen Untersuchungen von Radomír Pleiner zur Schmiedetechnologie im Frühmittelalter des mährischen Raumes. ${ }^{71}$ Später befasste sich Herbert Westphal ebenfalls mit der Technologie frühmittelalterlicher Waffen, besonders der Saxe, wobei er seinen Fokus auf den nordwestdeutschen Raum legte. ${ }^{72}$

Nach Pleiner sind die metallografischen Parameter für die Beschreibung von Eisenobjekten folgende: $:^{73}$

- Innerer Aufbau (Metallgefüge, Schweißnähte),

- Verunreinigungen mit nichtmetallischen Einschlüssen in verschiedenen Zonen,

- Härte des Metalls,

- chemische Zusammensetzung des Materials.

\subsection{Probenentnahme, Aufbereitung und Analyse}

Bei jedem Sax wurden zwei versetzte Proben vom Schneiden-sowie Rückenmaterial, die knapp über die Klingenmitte reichen, mit einer Laubsäge entnommen (Abb. 3-6). Das Probenmaterial wurde in Epoxidharz gegossen und Querschliffe $^{74}$ angefertigt. Mittels einer Ätzung mit 3\% alkoholischer Salpetersäure (Nital) wurde das Sekundärgefüge (Kohlenstoffverteilung) erkenntlich gemacht. Nach der Analyse des Sekundärgefüges wurde die Ätzlösung nach Oberhoffer verwendet, wodurch das Primärgefüge (sonstige Legierungselemente) untersucht werden konnte. Die metallografischen Analysen wurden mit einem Auflichtmikroskop, im ungeätzten und geätzten Zustand, durchgeführt. Die Härtemessungen wurden mit einem VickersHärteprüfgerät vorgenommen.

$70 \mathrm{Zu}$ ersten technologischen Vergleichen mit anderen Saxen aus Oberösterreich siehe Abschnitt 6, Diskussion.

71 Pleiner 1967.

72 Westphal 1991. - Westphal 2002.

73 Pleiner 1970, 116.

74 Mit Diamantsuspension auf eine Korngröße von $1 \mu$ poliert.

\subsection{Metallografische Auswertungen}

\subsubsection{Langsax A-1775 \\ Schlacken}

Das Metall weist unterhalb der Schweißnaht meist horizontale Schlackenzeilen auf (Abb. 7, 8). Im Trägermaterial sind hauptsächlich senkrechte, zum Teil lange Schlackenzeilen erkennbar (Abb. 9). Das Metall weist einen mittleren Schlackengehalt auf.

\section{Korngröße (nach DIN 50601)}

Die Korngröße bei der Nitalätzung entspricht im Rückenmaterial, knapp oberhalb der Schweißnaht, dem Gefügebild (GB) Nr. VI, unterhalb der Schweißnaht (GB) Nr. I-IV und im Schneidenmaterial (GB) Nr. VII-VIII.

\section{Ätzung mit Nital}

Probe aus dem Rückenmaterial: Die Probe weist im Rückenmaterial grobkörnigen Ferrit auf (Abb. 13) und im rechten unteren Bereich sind einige Neumannbänder vorhanden.

Probe aus dem Schneidenmaterial: Das Schneidenmaterial weist einen höheren Kohlenstoffanteil als das Rückenmaterial auf (Abb. 11). Im mittleren Teil der Probe ist eine Schweißnaht zu erkennen, die annähernd horizontal ist bzw. leicht nach rechts hin abfällt (Abb. 7). Knapp oberhalb der Schweißnaht ist ferritisch-perlitisches Gefüge zu erkennen, da Kohlenstoff vom Schneidenmaterial in das Trägermaterial diffundiert ist (Abb. 12). Unterhalb der Schweißnaht, am rechten Rand der Probe, ist in einem kleinen Bereich wiederum ein unvollständiges Härtegefüge (Bainit?) vorhanden (Abb. 7). An der Schneide befindet sich unvollständiges Härtegefüge (Abb. 10), wobei es sich aufgrund des niederen Härtewerts von 380 (HV) auch um feinststreifigen Perlit handeln könnte. Oberhalb der Schneide ist Widmannstättengefüge mit teilweise perlitisch-ferritischem Gefüge zu erkennen.

\section{Ätzung nach Oberhoffer (Aufbau)}

Der Sax wurde aus zwei Metallteilen zusammengesetzt, einem Rücken- und einem Schneidenteil (Abb. 8, 9).

\subsubsection{Langsax $A-1776$}

\section{Schlacken}

Das Trägermaterial weist einen hohen Schlackenanteil auf (Abb. 16). Es lassen sich grob eingeformte Schlackenzeilen erkennen. Das Schneidenmaterial weist im Gegensatz zum Trägermaterial einen geringen Schlackenanteil auf (Abb. 14, 15). 
Korngröße (nach DIN 50601)

Die Korngröße bei der Nitalätzung entspricht im Trägermaterial den Gefügebildern (GB) Nr. I-II. Im linken Teil des Trägermaterials ist das Gefüge bedeutend feinkörniger und entspricht den Gefügebildern (GB) Nr. VII-VIII. Das Schneidenmaterial weist ein sehr feinkörniges Gefüge auf und entspricht dem Gefügebild (GB) Nr. VIII.

\section{Ätzung mit Nital}

Probe aus dem Rückenbereich: Im rechten Teil der Probe ist grobkörniges ferritisches Gefüge vorhanden. An der linken Seite befindet sich feinkörniges ferritisches Gefüge mit Korngrenzen-Zementit bzw. -Perlit (Abb. 20).

Probe aus dem Schneidenmaterial: Im vorderen Teil der Schneide (Abb. 17) befindet sich ein Härtegefüge (Martensit) mit Härtewerten von über 750 (HV). Darüber befindet sich unvollständiges Härtegefüge (Bainit), das zur Schweißnaht hin in perlitisches Gefüge übergeht (Abb. 14). Die Schweißnaht verläuft schräg von links oben nach rechts unten und zieht dann wieder v-förmig nach oben (Abb. 14-15). Über die Schweißnaht ist Kohlenstoff in das Trägermaterial diffundiert (Abb. 18, 19).

\section{Ätzung nach Oberhoffer (Aufbau)}

Der Sax wurde aus zwei Metallteilen zusammengesetzt, einem Rücken- und einem Schneidenteil (Abb. 15, 16).

\subsubsection{Leichter Breitsax A-1783}

\section{Schlacken}

Das Metall weist im Schneiden- sowie im Trägermaterial einen geringen Schlackenanteil auf (Abb. 21-23). Entlang der querverlaufenden Schweißnaht, zwischen Träger- und Schneidenmaterial, sind einige Schlacken $\mathrm{zu}$ erkennen (Abb. 21, 22).

\section{Korngröße (nach DIN 50601)}

Die Korngröße bei der Nitalätzung entspricht im Rückenmaterial den Gefügebildern (GB) Nr. I-IV. Im Schneidenmaterial entspricht die Korngröße den Gefügebildern (GB) Nr. V-VIII.

\section{Ätzung mit Nital}

Probe aus dem Rückenmaterial: Die Probe weist sehr homogenes grobkörniges ferritisches Gefüge mit sehr wenig Schlacken auf.

Probe aus dem Schneidenmaterial: Im Schneidenmaterial ist hauptsächlich unvollständiges Härtegefüge (Bainit) und in einigen Bereichen perlitisch-ferritisches Gefüge zu erkennen (Abb. 24-26). Es sind zwei Schweißnähte festzustellen, wobei die untere bereits im Barren vorhanden war (Abb. 27). Die obere, querverlaufende Schweißnaht mit Schlackeneinschlüssen stellt dagegen eine intentionelle Verbindung zwischen weicherem Träger- und hartem Schneidenmaterial dar. Vom Schneidenmaterial ist Kohlenstoff über die Schweißnaht in das Trägermaterial diffundiert, daher ist ferritisch-perlitisches Gefüge knapp oberhalb der Naht zu erkennen (Abb. 21).

\section{Ätzung nach Oberhoffer (Aufbau)}

Der Sax wurde aus zwei Metallteilen zusammengesetzt, einem Rücken- und einem Schneidenteil (Abb. 22, 23).

\subsubsection{Langsax A-1784}

\section{Schlacken}

Im Trägermaterial sind einige grob eingeformte Schlackenzeilen zu erkennen (Abb. 30). Die Probe aus dem Rückenmaterial weist einen mittleren bis hohen Schlackenanteil auf. Das Schneidenmaterial hat hingegen einen geringen Schlackenanteil (Abb. 28, 29).

\section{Korngröße (nach DIN 50601)}

Die Korngröße bei der Nitalätzung differiert im Rückenmaterial aufgrund der inhomogenen Kohlenstoffverteilung zwischen den Gefügebildern (GB) Nr. III-VII. Im Schneidenmaterial entspricht die Korngröße den Gefügebildern (GB) Nr. VII-VIII.

\section{Ätzung mit Nital}

Probe aus dem Rückenmaterial: Das Trägermaterial weist hauptsächlich ferritisch-perlitisches Gefüge auf. Ferrit ist in den beiden Ecken links oben und links unten zu erkennen. In der Mitte der Probe ist perlitisches Gefüge vorhanden.

Probe aus dem Schneidenmaterial: Im untersten Teil der Schneide ist unvollständiges Härtegefüge (Martensit mit geringen Bainitanteilen) vorhanden (Abb. 31, 32). Zum Rückenmaterial hin geht es in perlitisches Gefüge über (Abb. 28). In der Probe sind drei Schweißnähte als helle Linien zu erkennen. Während die oberen beiden querverlaufenden Nähte intentionelle Verbindungen darstellen (Abb. 34), war die untere u-förmige Naht bereits im Barren vorhanden (Abb. 33).

\section{Ätzung nach Oberhoffer (Aufbau)}

Der Sax wurde aus drei Metallstäben zusammengesetzt, einem Rücken-, einem Mittel- und einem Schneidenteil (Abb. 29, 30). 


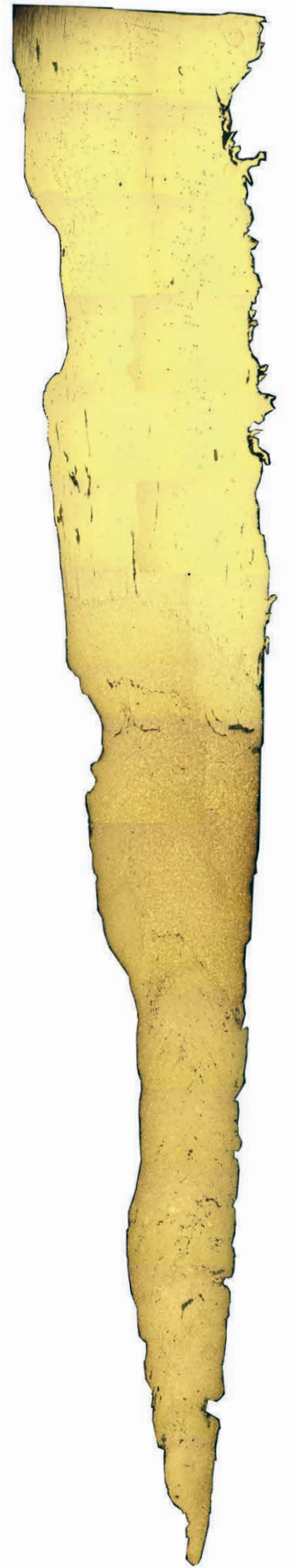

Abb. 7. Überackern-Langsax A-1775, Übersicht des Schneidenmaterials, Ätzung mit Nital.

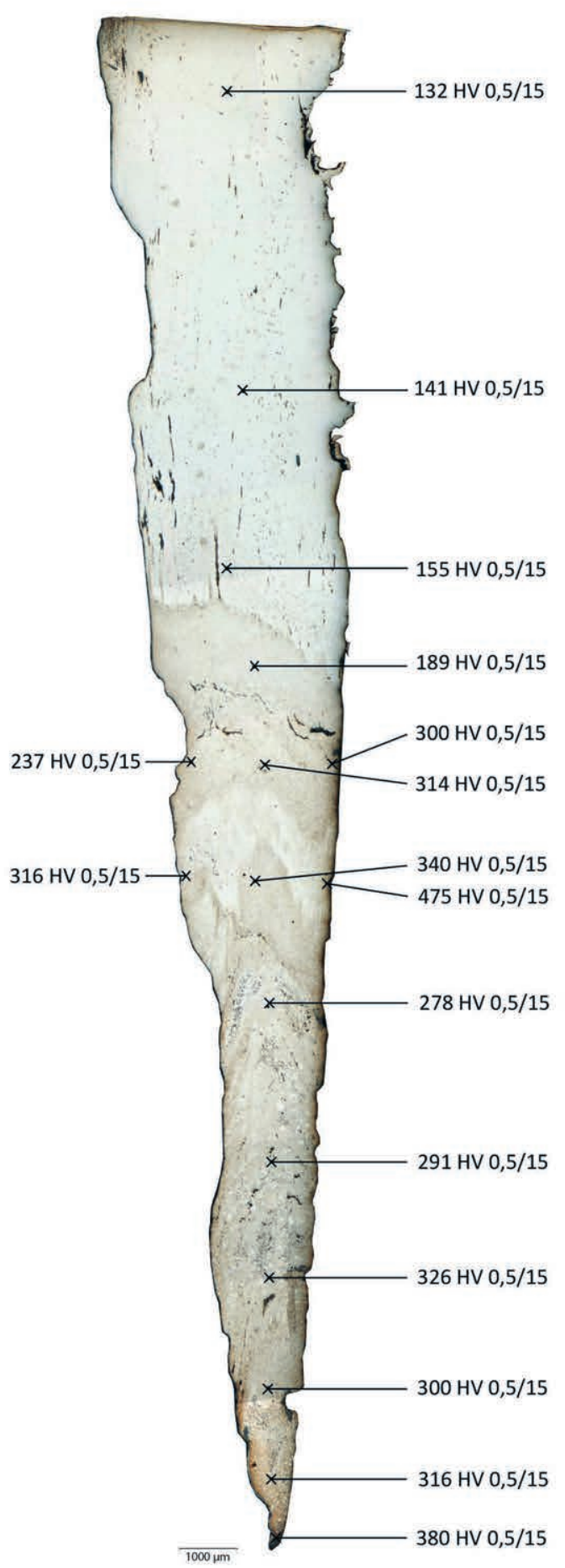

Abb. 8. Überackern-Langsax A-1775, Übersicht des Schneidenmaterials, Ätzung nach Oberhoffer mit Härtewerten nach Vickers (HV). 

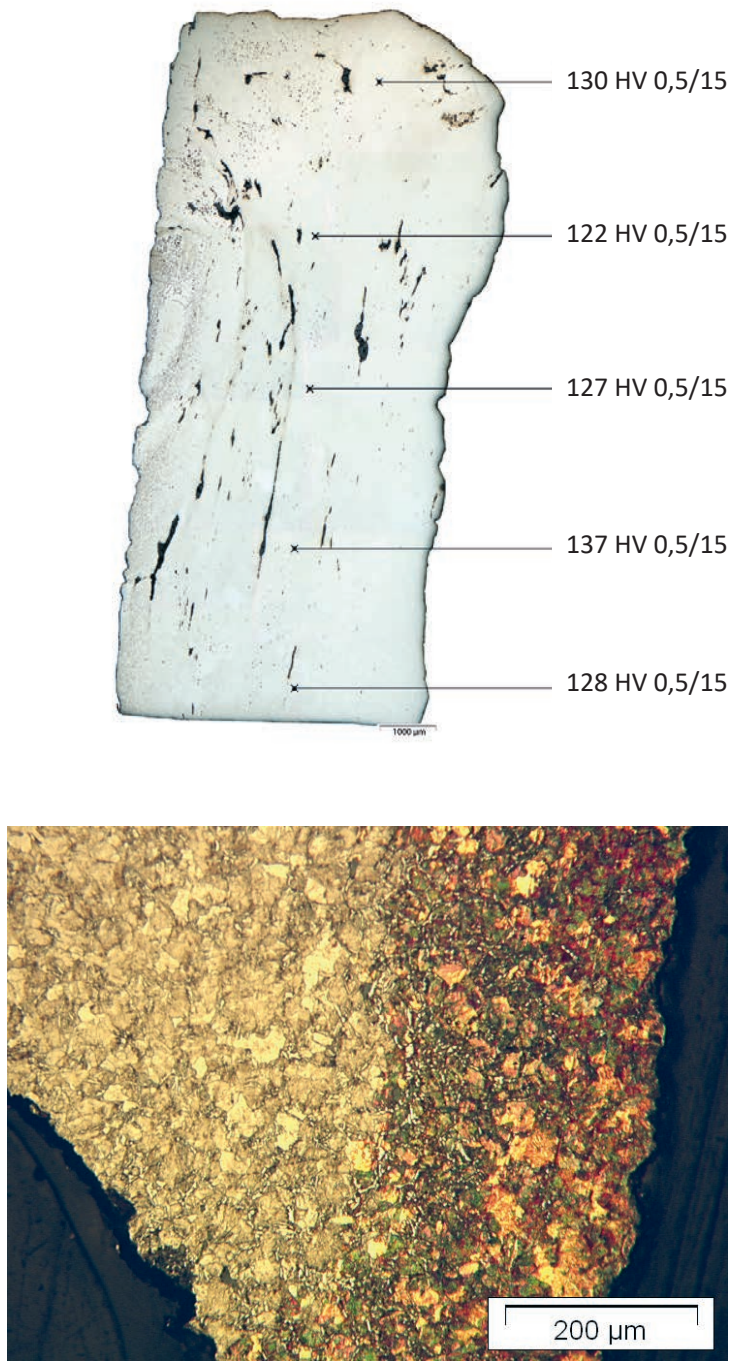

Abb. 10. Unteres Schneidenmaterial mit unvollständigem Härtegefüge (Martensit, Bainit) im rechten und perlitischem Gefüge im linken Bereich.

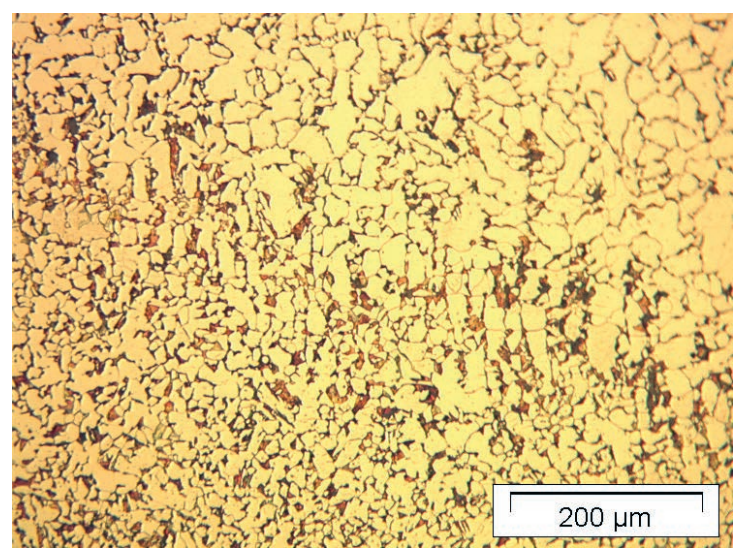

Abb. 12. Übergang vom Schneiden- zum Rückenteil, mit perlitisch-ferritischem Gefüge unterhalb und Ferrit mit Korngrenzenperlit oberhalb der Schweißnaht.
Abb. 9. Überackern-Langsax A-1775, Übersicht des Rückenmaterials, Ätzung nach Oberhoffer mit Härtewerten nach Vickers (HV).

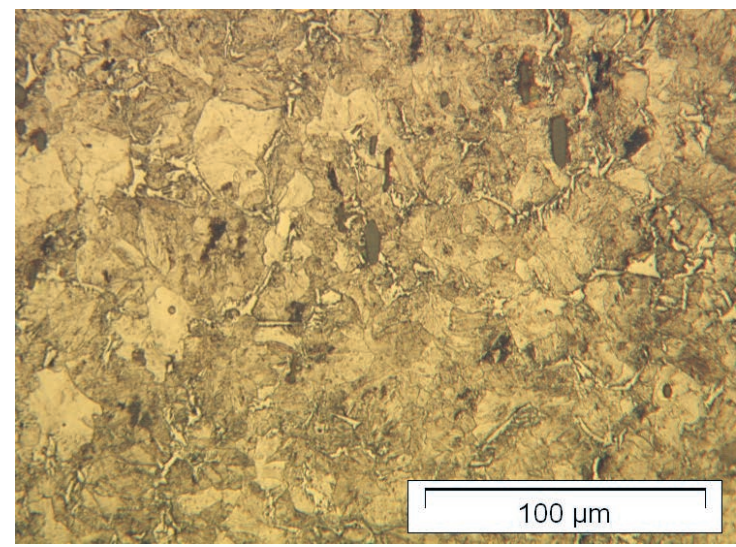

Abb. 11. Perlit mit Korngrenzenferrit im zentralen Schneidenbereich.

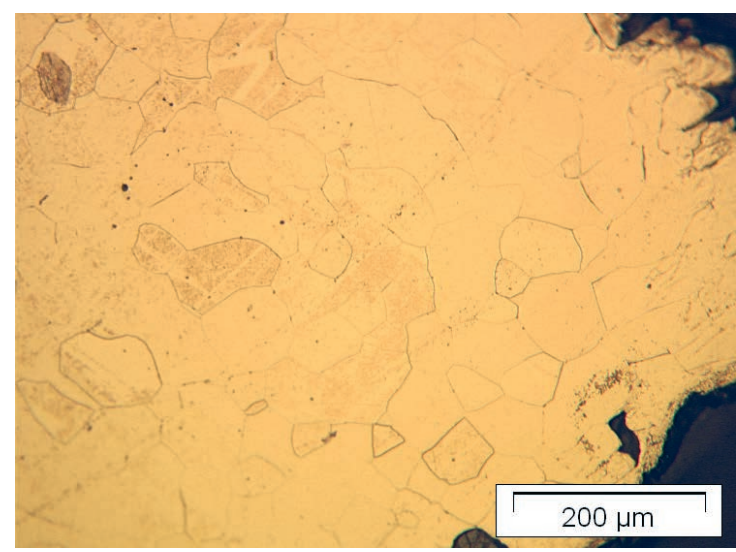

Abb. 13. Grobkörniger Ferrit im Rückenmaterial. 


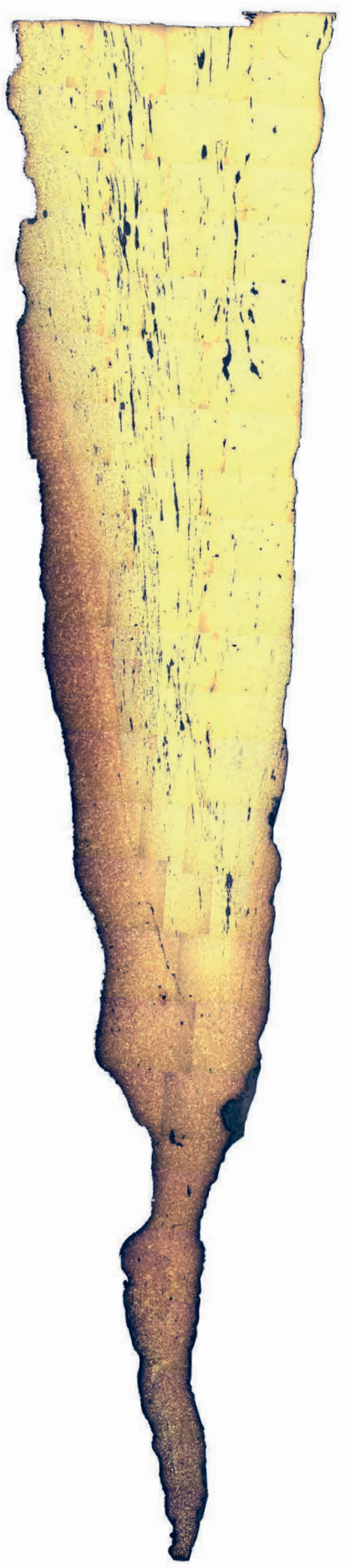

Abb. 14. Überackern-Langsax A-1776, Übersicht des Schneidenmaterials, Ätzung mit Nital.

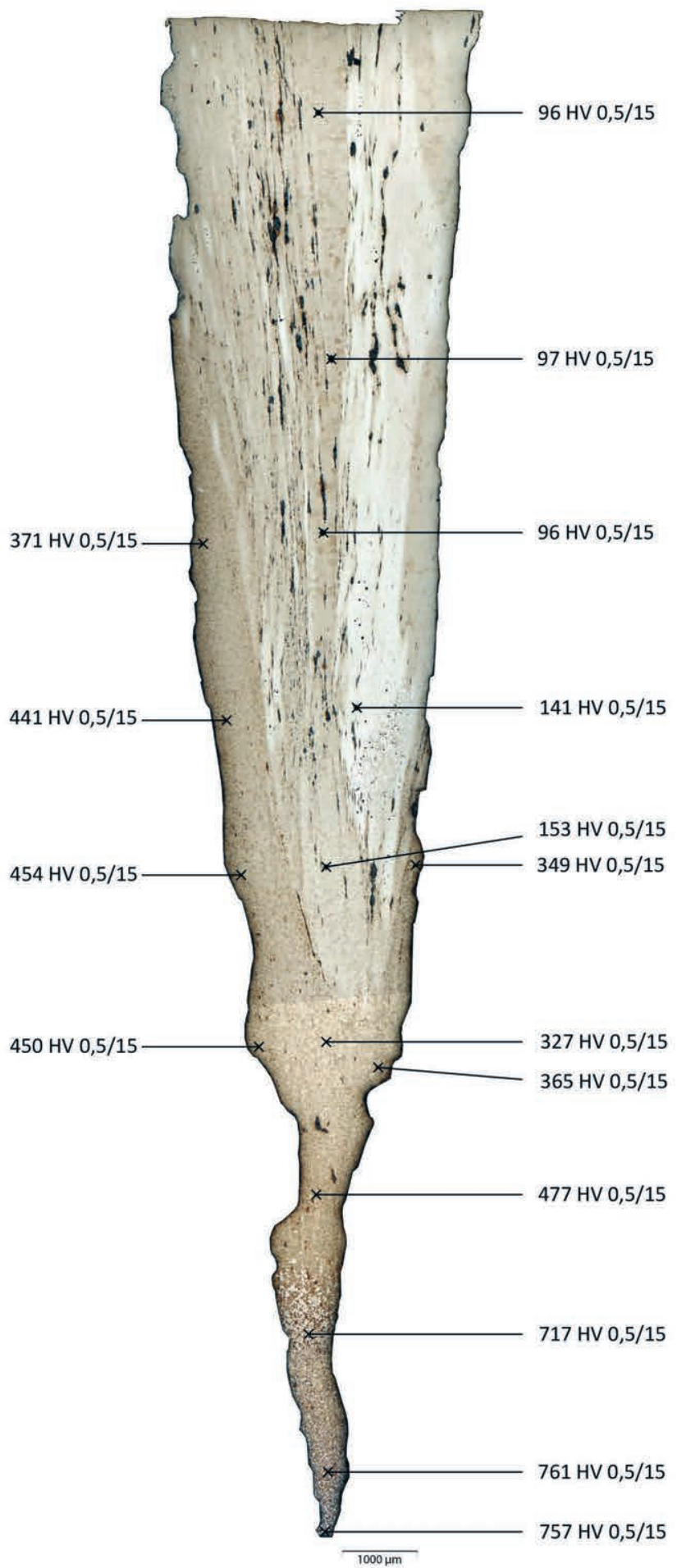

Abb. 15. Überackern-Langsax A-1776, Übersicht des Schneidenmaterials, Ätzung nach Oberhoffer mit Härtewerten nach Vickers (HV). 

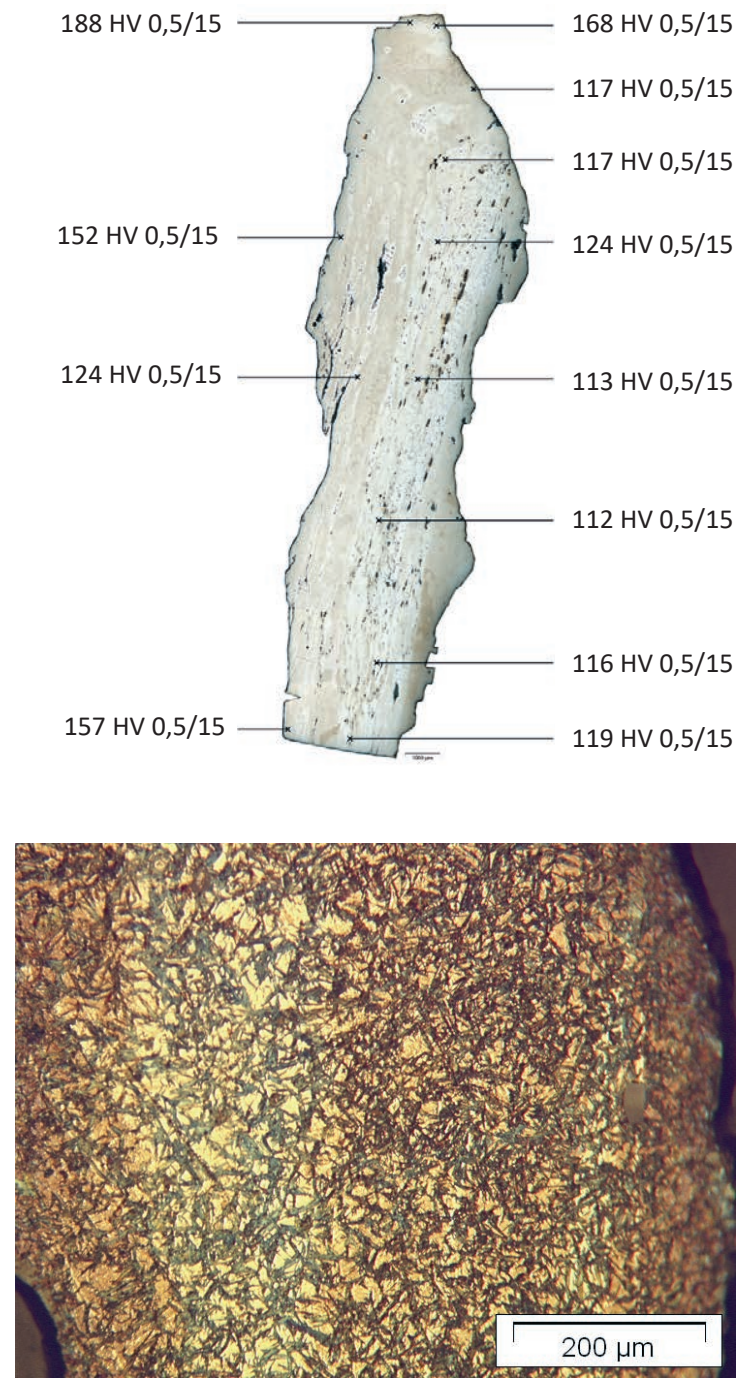

Abb. 17. Unvollständiges Härtegefüge (Martensit) im unteren Schneidenbereich.

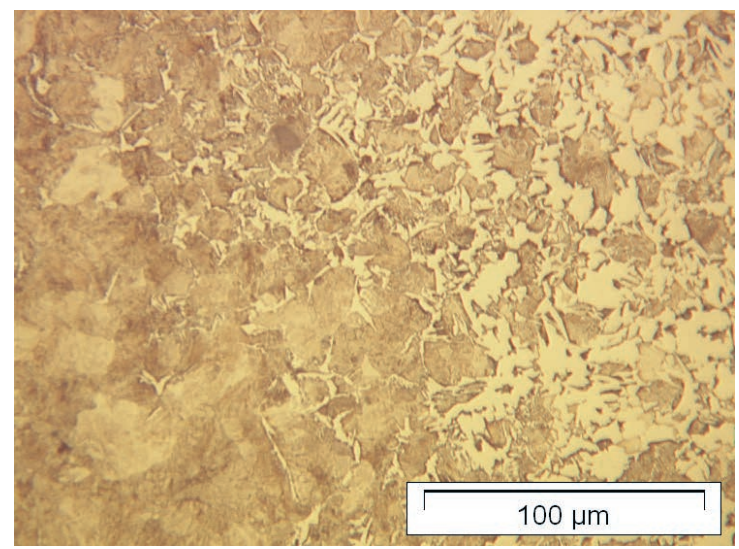

Abb. 19. Detailaufnahme des Übergangs vom perlitischen Gefüge des Schneidenmaterials (links) zum ferritisch-perlitischen Gefüge im Rückenmaterial (rechts).
Abb. 16. Überackern-Langsax A-1776, Übersicht des Rückenmaterials, Ätzung nach Oberhoffer mit Härtewerten nach Vickers (HV).

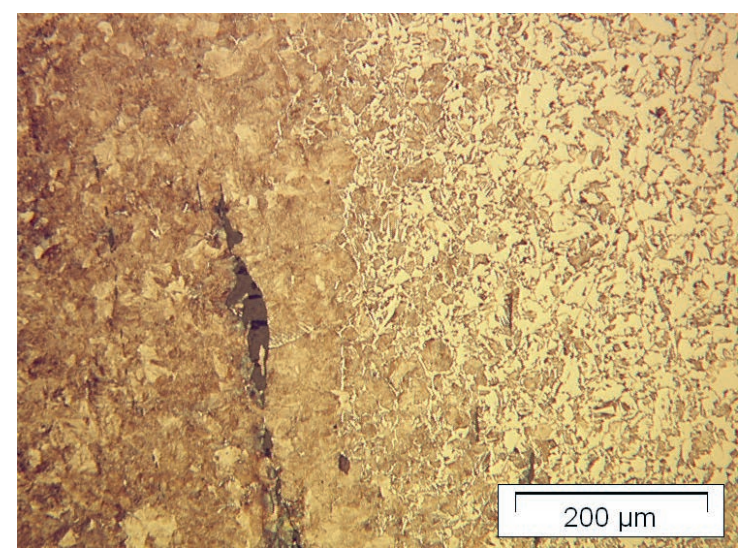

Abb. 18. Übergang vom perlitischen Gefüge des Schneidenmaterials (links) zum ferritisch-perlitischen Gefüge im Rückenmaterial (rechts).

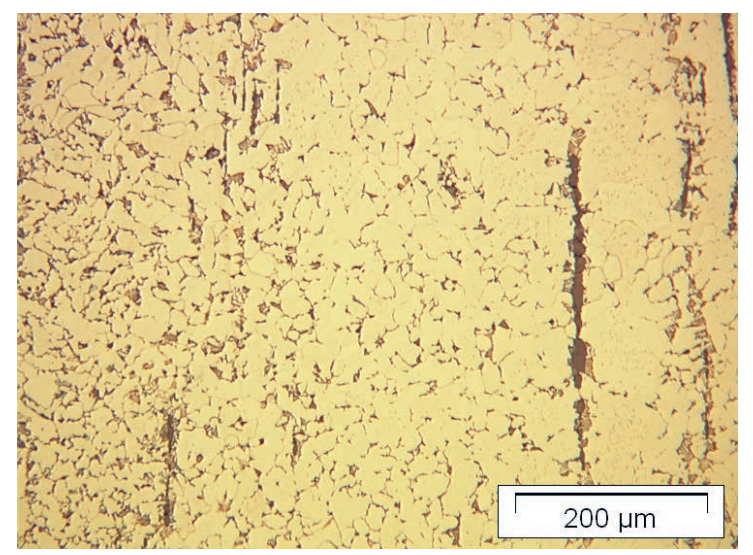

Abb. 20. Ferrit mit Korngrenzenperlit und zeilenförmig eingestreckten Schlacken im Rückenmaterial. 

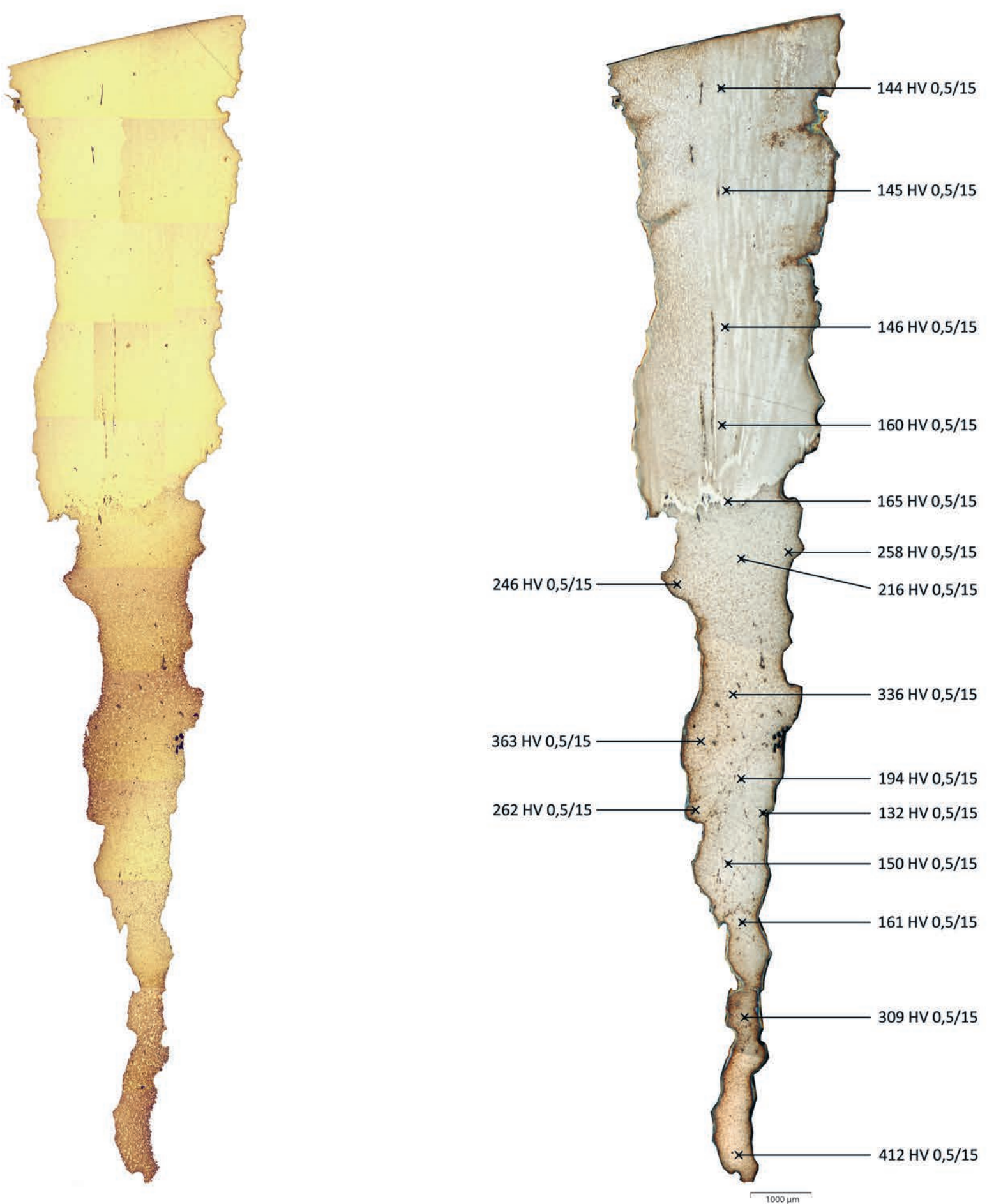

Abb. 21. Überackern-leichter Breitsax A-1783, Übersicht des Schneidenmaterials, Ätzung mit Nital.

Abb. 22. Überackern-leichter Breitsax A-1783, Übersicht des Schneidenmaterials, Ätzung nach Oberhoffer mit Härtewerten nach Vickers (HV). 

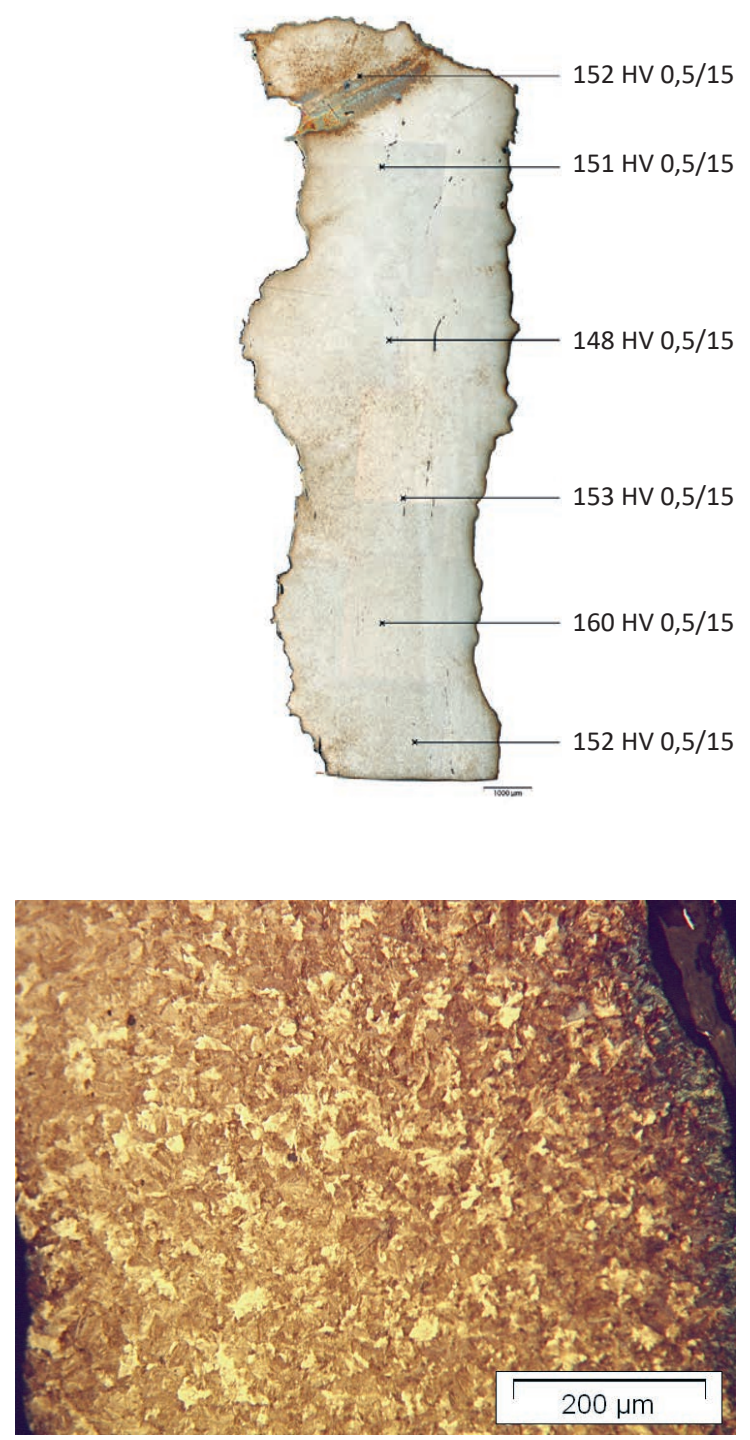

Abb. 24. Unterer Schneidenbereich mit unvollständigem Härtegefüge (Bainit).

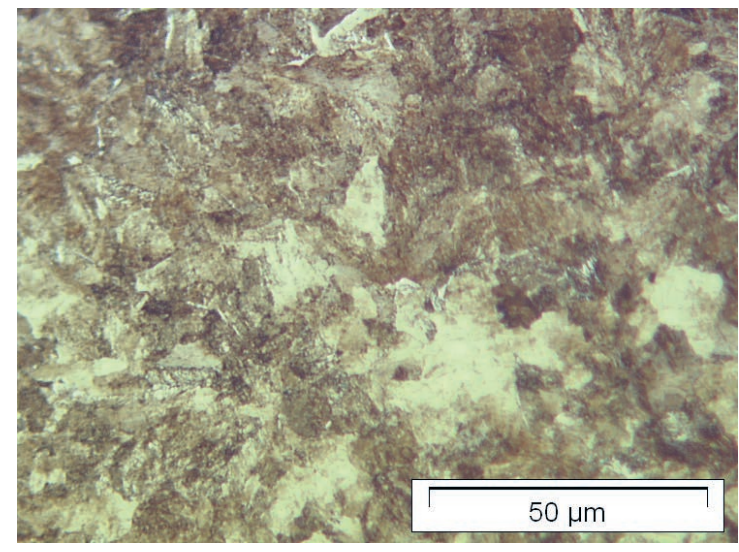

Abb. 26. Detailaufnahme des Perlits im oberen Schneidenbereich.
Abb. 23. Überackern-leichter Breitsax A-1783, Übersicht des Rückenmaterials, Ätzung nach Oberhoffer mit Härtewerten nach Vickers (HV).

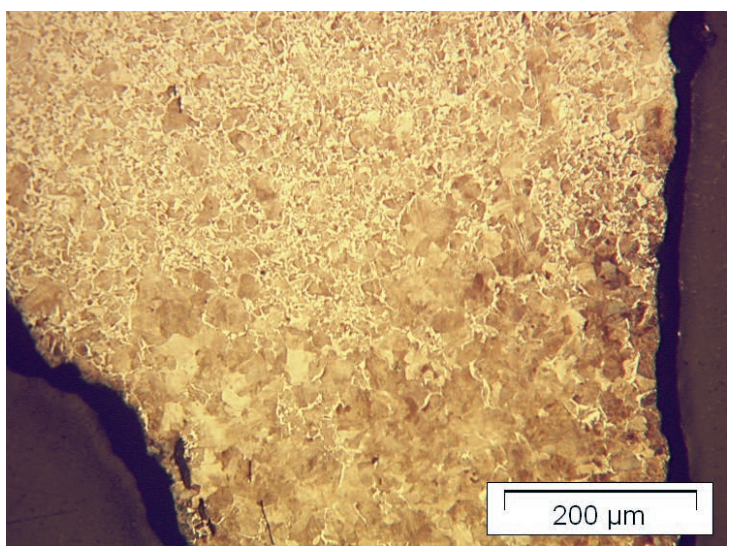

Abb. 25. Übergang vom perlitischen zum perlitisch-ferritischen Gefüge im oberen Schneidenbereich.

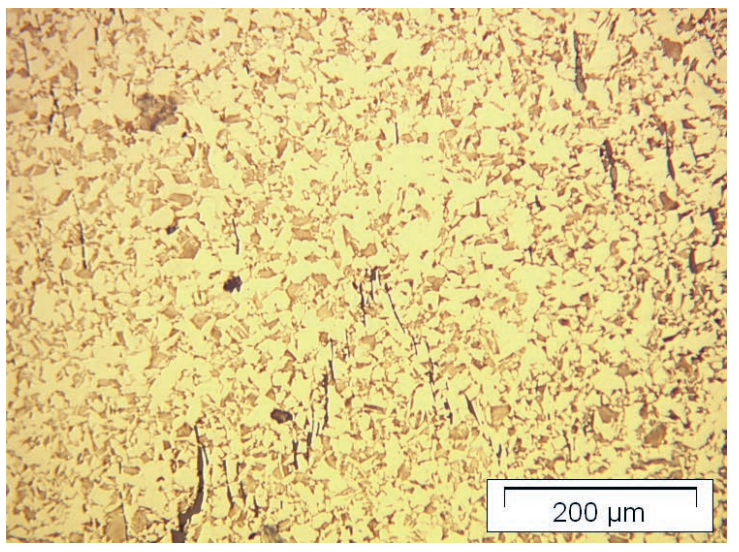

Abb. 27. Ferrit mit Korngrenzenperlit und Schlackeneinschlüssen entlang der unteren Schweißnaht im Schneidenmaterial. 


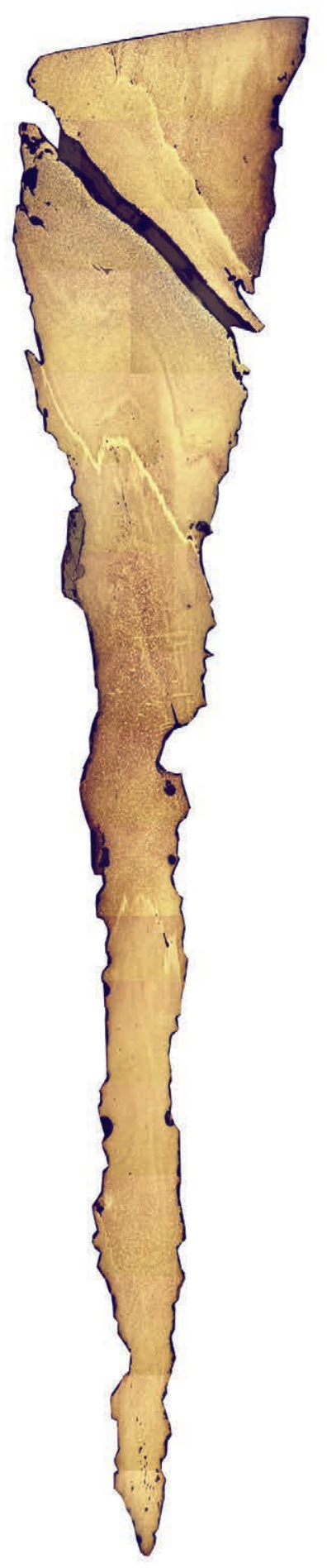

Abb. 28. Überackern-Langsax A-1784, Übersicht des Schneidenmaterials, Ätzung mit Nital.

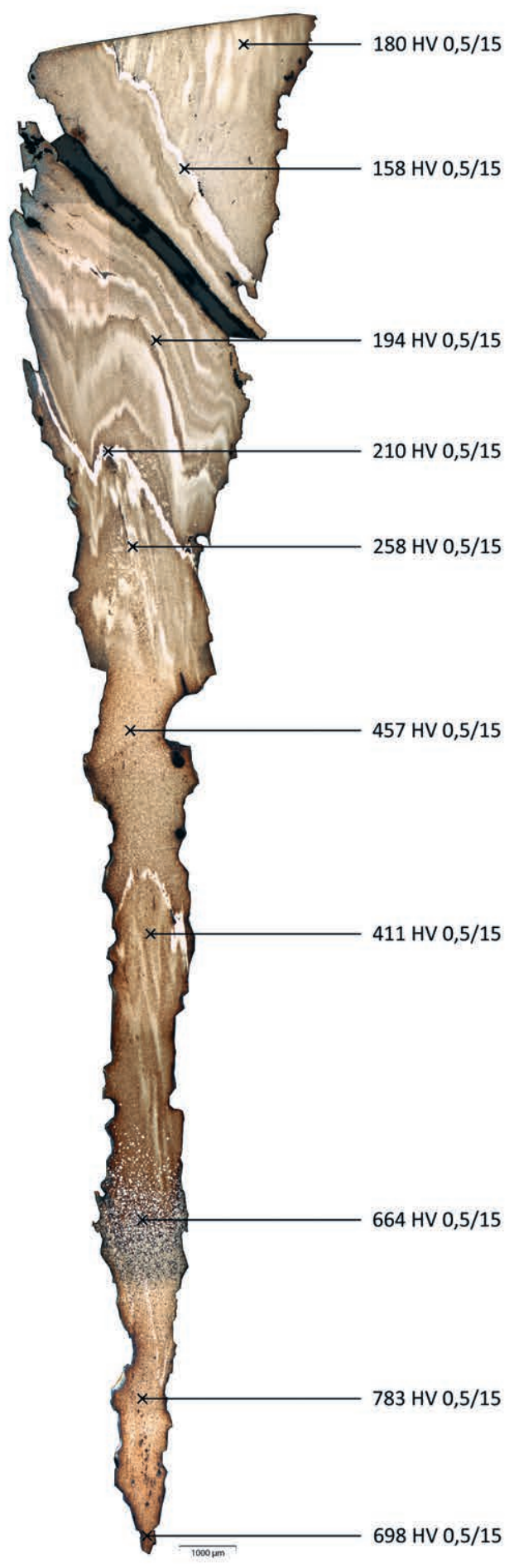

Abb. 29. Überackern-Langsax A-1784, Übersicht des Schneidenmaterials, Ätzung nach Oberhoffer mit Härtewerten nach Vickers (HV). 

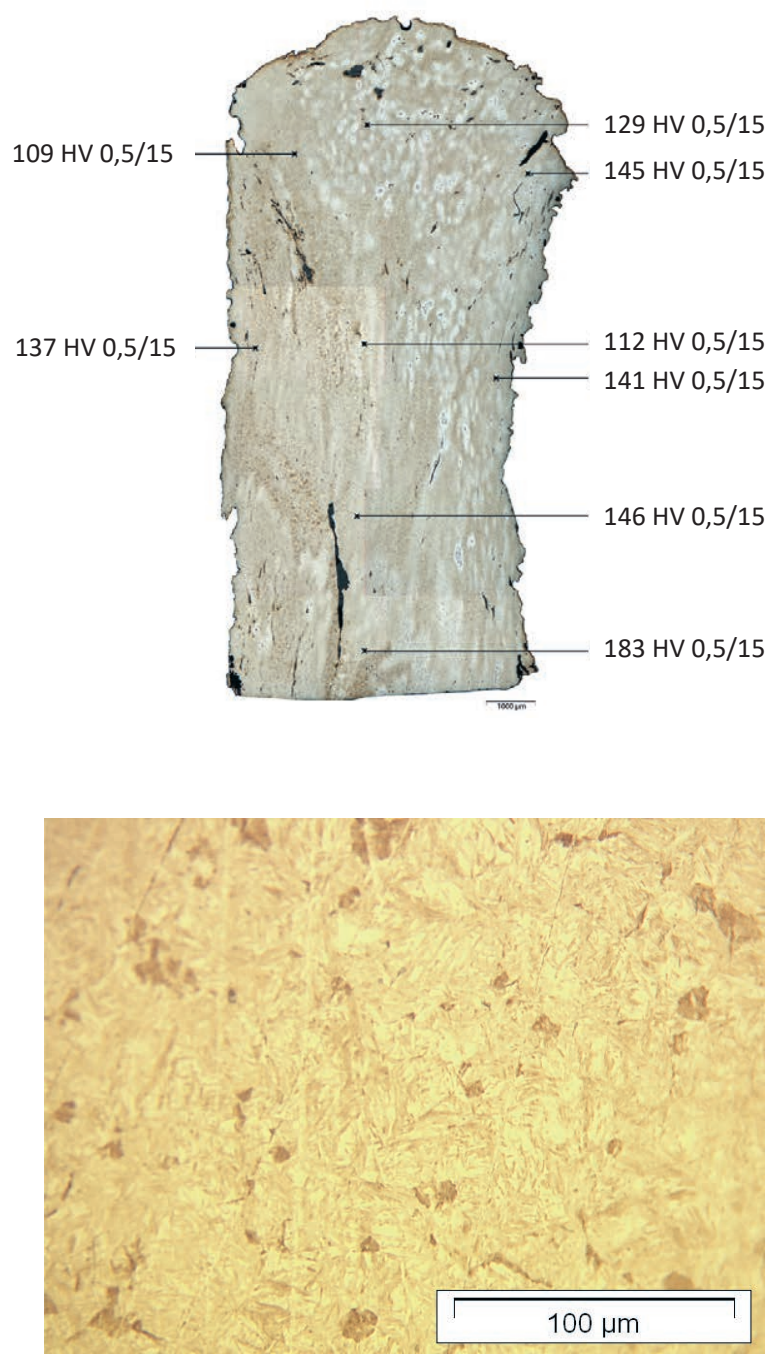

Abb. 31. Unvollständiges Härtegefüge (Martensit mit geringen Bainitanteilen) im unteren Schneidenbereich.

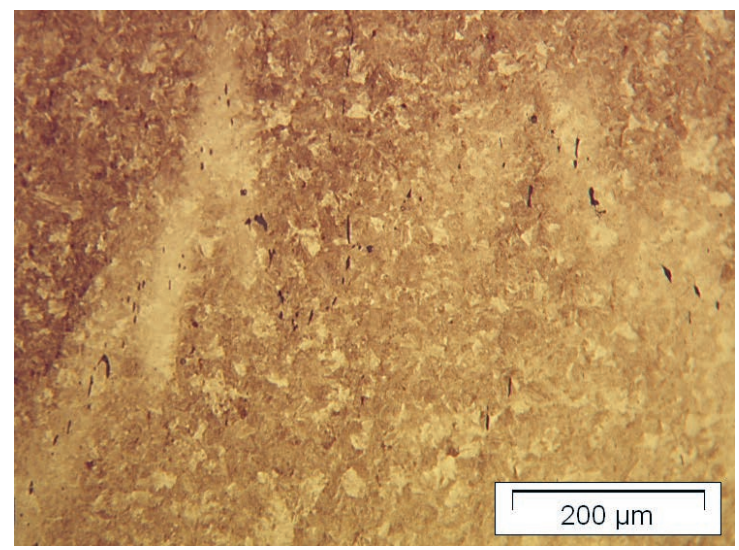

Abb. 33. U-förmige Schweißnaht mit Schlackeneinschlüssen im zentralen Schneidenbereich, perlitisches Gefüge oberund unterhalb der Schweißnaht.
Abb. 30. Überackern-Langsax A-1784, Übersicht des Rückenmaterials, Ätzung nach Oberhoffer mit Härtewerten nach Vickers (HV).

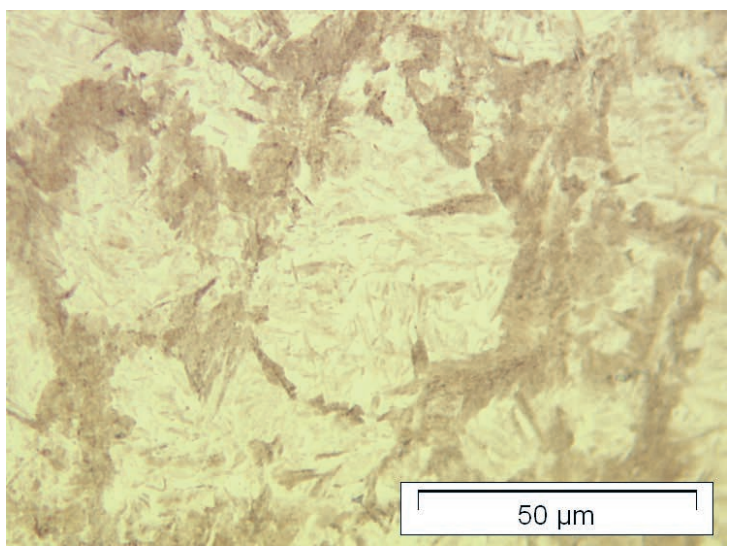

Abb. 32. Detailaufnahme des Martensits im Schneidenteil.

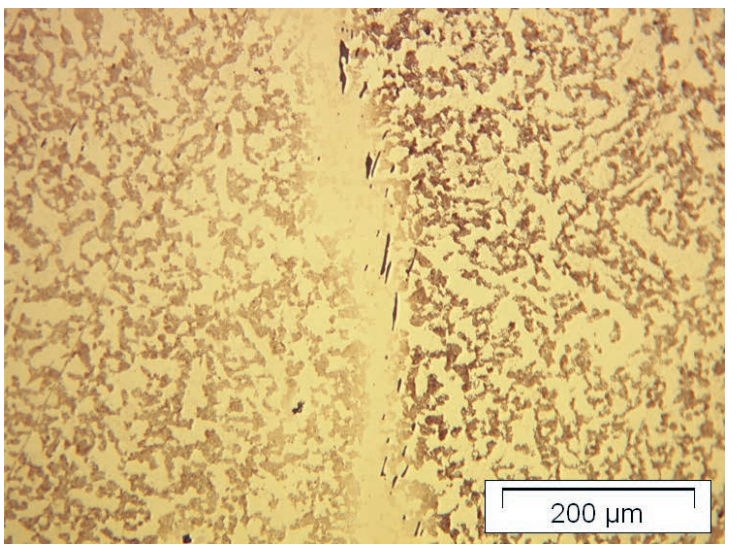

Abb. 34. Ferrit mit Korngrenzenperlit am Übergang vom Schneiden- zum Rückenteil, mit Schlackeneinschlüssen entlang der Schweißnaht. 


\subsection{Interpretation}

Drei der vier Saxe aus Überackern sind zweiteilig aufgebaut, während der Langsax A-1784 aus mindestens drei Teilen zusammengesetzt wurde. Sie weisen allesamt Schweißnähte auf, wobei das Schneidenmaterial unterhalb der Nähte bei allen vier Stücken einen höheren Kohlenstoffgehalt aufweist als das darüber liegende Rückenmaterial. Letzteres besteht bei allen beprobten Exemplaren hauptsächlich aus grobkörnigem Ferrit, während das Schneidenmaterial durchwegs Perlit aufweist. Dies bedeutet, dass bei allen vier Saxen harter, kohlenstoffhaltiger Stahl an elastisches, kohlenstoffarmes Eisen angeschweißt wurde.

Unter dem Mikroskop weist das Schneidenmaterial des Langsaxes A-1775 perlitisch-ferritisches Gefüge sowie Widmanstättengefüge auf. Somit handelt es sich um kohlenstoffhaltigen Stahl. Es ist anzunehmen, dass der Schmied versucht hat, das Material zu härten. An der Schneidenkante wurde ein Härtewert von 380 (HV) gemessen. Dabei könnte es sich um unvollständiges Härtegefüge handeln, was jedoch nicht eindeutig feststellbar ist. In einem kleinen Bereich unterhalb der Schweißnaht, am rechten Rand der Probe, ist eindeutig ein unvollständiges Härtegefüge (Bainit?) mit einem Härtewert von 475 (HV) zu erkennen.

Die Probe aus dem Schneidenmaterial des Langsaxes A-1776 weist im untersten Teil ein Härtegefüge (Martensit) mit Härtewerten von 717 bis 761 (HV) auf. Dieser Sax besitzt als einziger aus dem Fundensemble von Überackern martensitisches Gefüge. Dies bedeutet, dass an diesem Stück der Härtungsprozess auf gekonnte Art und Weise durchgeführt wurde. An der linken Seite der Probe des Rückenmaterials ist Korngrenzen-Zementit bzw. -Perlit vorhanden, weshalb dieser Teil der Probe höhere Härtewerte aufweist.

Der Härtewert von 412 (HV) im Schneidenbereich des leichten Breitsaxes A-1783 ist auf eine erhöhte Abkühlgeschwindigkeit zurückzuführen. Ausgeprägtes Härtegefüge konnte jedoch nicht festgestellt werden. Es sind zwei Schweißnähte zu erkennen. Die untere liegt im Schneidenmaterial und ist u-förmig (verkehrtes U). Dies ist darauf zurückzuführen, dass die Naht bereits im Barren vorhanden war und beim Ausschmieden bzw. Strecken des Materials diese Form annahm. Im mittleren Drittel der Probe ist eine querverlaufende Schweißnaht mit Schlackeneinschlüssen $\mathrm{zu}$ erkennen, welche durch die intentionelle Verbindung des Rücken- mit dem Schneidenmaterial entstanden ist. Das Rückenmaterial oberhalb des Schneidenbereiches bzw. der Schweißnaht besteht aus ferritischem Gefüge. Der Sax ist somit aus einem weichen Trägermaterial und kohlenstoffreichem (härtbarem) Schneidenmaterial zusammengesetzt.

Im Schneidenmaterial des Langsaxes A-1784 sind drei Schweißnähte sichtbar. Die erste von unten zu erkennende
Naht ist u-förmig (verkehrtes $U$ ) und war bereits im Barren vorhanden. Bei der zweiten von unten zu erkennenden Schweißnaht handelt es sich um eine bewusste Verbindung zweier kohlenstoffhaltiger Materialien. Die darüber liegende Naht stellt jene Verbindung dar, bei der das härtere, kohlenstoffreiche Schneidenmaterial an das weichere, kohlenstoffarme Trägermaterial angeschweißt wurde. Das unvollständige Härtegefüge (Martensit, Bainit) im unteren Teil der Probe weist darauf hin, dass das Material gehärtet wurde. Der besonders hohe Härtewert von 783 (HV) im unteren Schneidenmaterial ist wahrscheinlich auf vereinzelte Martensit-Inseln zurückzuführen.

\section{Diskussion}

Das Fundmaterial aus dem Gräberfeld von Überackern datiert in das 7. und frühe 8. Jh., wobei der Schwerpunkt im letzten Drittel des 7. und beginnenden 8. Jhs. liegt. Drei der vier Saxe des Fundensembles stammen aus dieser Phase. Das älteste Exemplar - der leichte Breitsax A-1783 - stammt hingegen aus der Zeit vor der Mitte des 7. Jhs., während der jüngste Sax - der Langsax A-1784 - im frühen 8. Jh. gefertigt wurde. Es ist daher bemerkenswert, dass alle vier Waffen deutliche Parallelen in ihrer Herstellungstechnik aufweisen. Sowohl beim leichten Breitsax als auch bei den drei Langsaxen wurde Schneidenmaterial aus Stahl an Rückenmaterial aus Eisen angeschweißt.

Insgesamt liegen die metallografischen Ergebnisse von neun Saxen aus Oberösterreich vor. Neben den vier Exemplaren aus Überackern wurden die Saxe aus Auhof, ${ }^{75}$ Gusen, ${ }^{76}$ Enns-Lauriacum, ${ }^{77}$ Bad Wimsbach-Neydharting ${ }^{78}$ und Gunskirchen-Moostal ${ }^{79}$ untersucht. Vergleicht man die Ergebnisse dieser Waffen, so zeigt sich, dass bei der Mehrheit der untersuchten Saxe verschiedene Materialien mit unterschiedlichen Eigenschaften durch Feuerverschweißen verbunden wurden. Kohlenstoffreiches Schneidenmaterial wurde an kohlenstoffarmes Rückenmaterial bei den Saxen aus Überackern, Auhof, Gusen und Enns angeschweißt. Lediglich die Saxe aus Bad Wimsbach und Gunskirchen wurden aus einem Stück geschmiedet.

Ein weiteres Verfahren, das zum technologischen Standard des 7. und frühen 8. Jh. gehörte, ist das Härten von

75 Szameit, Mehofer 2002, 138 und Abb. 6/A; 139, 152 und Abb. 7; 153 und Abb. 8; 146-147.

76 Szameit, Mehofer 2002, 138 und Abb. 6/B; 139-140, 154 und Abb. 9; 155 und Abb. 10; 146-147.

77 Szameit, Mehofer 2002, 138 und Abb. 6/C; 140, 156 und Abb. 11; 157 und Abb. 12; 146-147.

78 Nau 2016, 502-504 und Taf. 10-18; 539 und Abb. 1-6; 540 und Abb. 1-6.

79 SкомоRowski 2016, 549-550, 559-567 und Taf. 8-16. 
Klingen durch Erhitzen auf Rot- bzw. Weißglut und Abschrecken in Wasser oder speziellen Flüssigkeiten. ${ }^{80}$ Diese Form der Härtung wurde bei allen Klingen der neun untersuchten Saxe aus Oberösterreich nachgewiesen, wobei das Verfahren nicht in allen Fällen erfolgreich umgesetzt werden konnte. Während die Gruppe der erfolgreich gehärteten Waffen Härtewerte von 600-783 (HV) besitzen, weist die Gruppe der nur leicht gehärteten Klingen Werte zwischen 300 (HV) und max. 475 (HV) auf. Die höchsten Werte wurde im Schneidenmaterial der Saxe A-1784 und A-1776 aus Überackern mit max. 783 (HV) sowie 761 (HV) festgestellt. Daneben können die Saxe A-10103 aus Gusen und A-10217 aus Auhof mit Maximalwerten von $679(\mathrm{HV})^{81}$ sowie $661(\mathrm{HV})^{82} \mathrm{zu}$ der Gruppe der erfolgreich gehärteten Klingen gezählt werden. Die Saxe A-1775 (max. 380-475 HV) und A-1783 (max. 412) aus Überackern sowie A-8345 aus Enns (max. $425 \mathrm{HV}),{ }^{83} \mathrm{~A}-8115$ aus Bad Wimsbach (max. $370 \mathrm{HV})^{84}$ und A-8680 aus Gunskirchen (max. $\left.360 \mathrm{HV}\right)^{85}$ zählen zur Gruppe mit geringeren Härtewerten. Bei allen untersuchten Saxen aus Oberösterreich ist das ungehärtete kohlenstoffarme Rückenmaterial mit Werten von 100-190 (HV) deutlich weicher als das Schneidenmaterial.

Schließlich sei auf die unterschiedliche Reinheit des Schneiden- und Rückenmaterials der Langsaxe A-1776 Schneidenmaterial an verunreinigtes Trägermaterial angeschweißt. Die Reinheit des Schneidenmaterials und die hohe schmiedetechnologische Qualität dieser Exemplare verdeutlichen, dass die Verunreinigungen des Trägermaterials nicht auf die technologischen Standards der Werkstätten zurückzuführen sind. Die Produzenten dieser Langsaxe griffen auf Rohmaterialien mit unterschiedlicher Qualität zurück und achteten dabei darauf, hochwertiges Material für den primären Nutzungsbereich der Waffen - die Schneide-zu verwenden.

Zusammenfassend kann festgehalten werden, dass die Saxe aus Überackern dem technologischen Standard ihrer Zeit entsprachen und im Falle der Langsaxe A-1776 und A-1784 von hoher Qualität waren. und A-1784 hingewiesen. Bei diesen Waffen wurde reines

\section{Danksagung}

Für die Organisation und Betreuung der typologisch-chronologischen Untersuchungen der Saxe aus Überackern möchte ich Herrn Erik Szameit herzlich danken. Die metallografischen Analysen wurden gemeinsam mit Mathias Mehofer durchgeführt, dem ich für die Unterstützung und Erläuterungen danke. Schließlich möchte ich Herrn Bendeguz Tobias für weiterführende Hinweise meinen Dank aussprechen.

\section{Literatur}

BENINGER, KLOIBER 1962

E. Beninger, A. Kloiber, Oberösterreichs Bodenfunde aus baierischer und frühdeutscher Zeit, Jahrbuch des Oberösterreichischen Musealvereins 107, 1962, 125-250.

BÖHNER 1958

K. BÖHNER, Die fränkischen Altertümer des Trierer Landes. Germanische Denkmäler der Völkerwanderungszeit, Serie B: Die fränkischen Altertümer des Rheinlandes 1, Berlin 1958.

BRENNER 1912

E. Brenner, Der Stand der Forschung über die Kultur der Merowingerzeit, Bericht der Römisch-Germanischen Kommission 7, 1912, 253-351.

CHRISTLEIN 1966

R. Christlein, Das alamannische Reihengräberfeld von Marktoberdorf im Allgäu. Materialhefte zur Bayerischen Vorgeschichte 21, München 1966.

\section{CSAR 2002}

P. Csar, Das bajuwarische Gräberfeld von Rudelsdorf, Oberösterreich, Archaeologia Austriaca 86, 2002, 117-148.

CsIKY 2012

G. CsIKY, Saxe im awarenzeitlichen Karpatenbecken. In: T. VIDA (Hrsg.), Thesaurus Avarorum. Régészeti tanulmányok Garam Éva tiszteletére. / Archaeological Studies in Honour of Éva Garam. Budapest 2012, 371-393.

DAvidson 1962

H. E. Davidson, The Sword in Anglo-Saxon England: Its Archaeology and Literature. Oxford 1962.

ECKHART 1959

L. ECKHART, Bericht über die Notgrabung in Überackern 1958, Jahrbuch des Oberösterreichischen Musealvereins 104, 1959, 18-25.

Gregor von Tours, Libri historiarum decem

G. von Tours, Libri historiarum decem. In: B. KrusCh, W. Levison (Hrsg.), Scriptores rer. Merov. I/1: Gregorii Turonensis Opera 1. Monumenta Germaniae Historica, Hannover 1937-1951.

GRUNWALD 2002

L. Grunwald, Tote in Ruinen: Anmerkungen zu den frühmittelalterlichen Bestattungen des Moselmündungsgebietes in römischen Gebäuderesten, Acta Praehistorica et Archaeologica 34, 2002, 95-111.

HÄRKE 1992

H. HäRKE, Angelsächsische Waffengräber des 5. bis 7. Jahrhunderts. Zeitschrift für Archäologie des Mittelalters, Beiheft 6, Köln Bonn 1992.

HüBENER 1989

W. Hübener, Die Langsaxe der späten Merowingerzeit, Acta Praehistorica et Archaeologica 21, 1989, 75-84.

81 Szameit, Mehofer 2002, 146

82 Szameit, Mehofer 2002, 146.

83 Szameit, Mehofer 2002, 146.

84 Nau 2016, 533 und Taf. 13.

85 Sкомоrowski 2016, 561 und Taf. 10.
KARNITSCH 1933

P. KaRNITSCH, Ein Reihengräberfeld und römische Funde in Ueberackern. Der römische Limes in Österreich 17, Wien 1933, 145-162. 


\section{Kiss 2014}

A. P. Kiss, Huns, Germans, Byzantines? The origins of the narrow bladed long seaxes, Acta Archaeologica Carpathica 49, 2014, 111-144.

Косн 1977

U. Косн, Das Reihengräberfeld bei Schretzheim. Germanische Denkmäler der Völkerwanderungszeit A 13. Berlin 1977.

Косн 1982

U. Косн, Die fränkischen Gräberfelder von Bargen und Berghausen in Nordbaden. Forschungen und Berichte zur Vor- und Frühgeschichte in Baden-Württemberg 12, Stuttgart 1982.

KосH 1994

U. Косн, Drei Langsaxe aus Ostbayern, Bericht der Bayerischen Bodendenkmalpflege 34/35, 1994, 181-201.

Косн 2001

U. Косн, Das alamannisch-fränkische Gräberfeld bei Pleidelsheim. Forschungen und Berichte zur Vor- und Frühgeschichte in Baden-Württemberg 60, Stuttgart 2001.

KROHN 2013

N. Kroнn, Goldlahn in der Alamannia: Beispiele aus Dürbheim „Häuslesrain“ (Kreis Tuttlingen) und Lahr-Burgheim, St. Peter (Ortenaukreis), Bericht der Bayerischen Bodendenkmalpflege 53, 2013, 355-360.

LINDENSCHMIT 1880-1889

L. Lindenschmit, Die Alterthümer der merovingischen Zeit. Handbuch der deutschen Alterthumskunde 1, Braunschweig 18801889.

LOSERT 2003

H. LOSERT, Das frühmittelalterliche Gräberfeld von Altenerding in Oberbayern und die „Ethnogenese“ der Bajuwaren. In: H. LoSERT, A. Pletersski, Altenerding in Oberbayern: Struktur des frühmittelalterlichen Gräberfeldes und „Ethnogenese“ der Bajuwaren. Berlin - Bamberg - Ljubljana 2003, 5-499.

MARTIN 1993

M. Martin, Observations sur l'armement de l'époque mérovingienne précoce. In: F. VALLET, M. KAZANSKI (Hrsg.), L'armée romaine et les barbares du III ${ }^{\mathrm{e}}$ au VII ${ }^{\mathrm{e}}$ siècle. Mémoires publiées par l'Association Française d'Archéologie Mérovingienne 5, Rouen 1993, 395-409.

MARTIN 2000

M. Martin, Mit Sax und Gürtel ausgestattete Männergräber des 6. Jahrhunderts in der Nekropole von Kranj (Slowenien). In: R. Bratoz (Hrsg.), Slovenija in sisednje dežele med antiko in karolinško dobo. Začetki slovenske etnogeneze 1, Ljubljana 2000, 141-196.

Müssemeier et al. 2003

U. Müssemeier, E. Nieveler, R. Plum, H. Pöppelmann, Chronologie der merowingerzeitlichen Grabfunde vom linken Niederrhein bis zur nördlichen Eifel. Materialien zur Bodendenkmalpflege im Rheinland 15, Köln 2003.

NAU 2016

E. NAU, Das bajuwarische Gräberfeld von Bad WimsbachNeydharting: Archäologische und archäometrische Auswertung. In: J. Leskovar (Hrsg.), Frühmittelalter in Oberösterreich: Inventare aus den archäologischen Sammlungen des Oberösterreichischen Landesmuseums. Studien zur Kulturgeschichte von Oberösterreich 40, Linz 2016, 499-540.
NeufFer-MüLler 1966

C. NeUfFer-MüLler, Ein Reihengräberfriedhof in Sontheim an der Brenz (Kreis Heidenheim). Veröffentlichungen des Staatlichen Amtes für Denkmalpflege Stuttgart A 11, Stuttgart 1966.

NøRGÅRD JøRGENSEN 1999

A. NøRGÅRD JøRgensen, Waffen und Gräber: Typologische und chronologische Studien zu skandinavischen Waffengräbern 520/30 bis 900 n. Chr. Nordiske Fortidsminder B 17, Kopenhagen 1999.

OLSÉN 1954

P. Olsén, Valsgärdestudien 2: Die Saxe von Valsgärde. Acta Musei Antiquitatum Septentrionalium Regiae Universitatis Upsaliensis 3, Uppsala 1945.

Pleiner 1967

R. Pleiner, Die Technologie des Schmiedes in der Großmährischen Kultur, Slovenska Archaeologica 15/1, 1967, 77-188.

Pleiner 1970

R. Pleiner, Zur Schmiedetechnik im römerzeitlichen Bayern, Bayerische Vorgeschichtsblätter 35, 1970, 113-142.

Siegmund 1996

F. Siegmund, Kleidung und Bewaffnung der Männer im östlichen Frankenreich. In: A. Wieczorek, P. Périn, K. v. Welck, W. Menghin (Hrsg.), Die Franken - Wegbereiter Europas, vor 1500 Jahren: König Chlodwig und seine Erben (Ausstellungskatalog, Mannheim, Paris, Berlin). Mainz 1996, 691-706.

SIEGMUND 1998

F. SIEGMUND, Merowingerzeit am Niederrhein: Die frühmittelalterlichen Funde aus dem Regierungsbezirk Düsseldorf und dem Kreis Heinsberg. Rheinische Ausgrabungen 34, Köln 1998.

SкOMOROWSKi 2016

R. Sкомовошsкi, Die frühmittelalterlichen Funde aus Gunskirchen-Moostal. In: J. Leskovar (Hrsg.), Frühmittelalter in Oberösterreich: Inventare aus den archäologischen Sammlungen des Oberösterreichischen Landesmuseums. Studien zur Kulturgeschichte von Oberösterreich 40, Linz 2016, 541-568.

Szameit 1987

E. SzAmeIT, Karolingerzeitliche Waffenfunde aus Österreich, Teil II: Die Saxe und Lanzenspitzen, Archaeologia Austriaca 71, 1987, 155-171.

SZAmeit, Mehofer 2002

E. Szameit, M. Mehofer, Technologische Untersuchungen an Waffen des Frühmittelalters aus Oberösterreich, Jahrbuch des Oberösterreichischen Musealvereins 147/I, 2002, 127-168.

WERNARD 1998

J. WERNARD, „Hic scramasaxi loquuntur“: Typologisch-chronologische Studie zum einschneidigen Schwert der Merowingerzeit in Süddeutschland, Germania 76/2, 1998, 747-787.

Westphal 1991

H. Westphal, Untersuchungen an Saxklingen des sächsischen Stammesgebietes: Schmiedetechnik, Typologie, Dekoration. Studien zur Sachsenforschung 7, Hildesheim 1991, 271-365.

WestPHAL 2002

H. Westphal, Franken oder Sachsen? Untersuchungen an frühmittelalterlichen Waffen. Studien zur Sachsenforschung 14, Oldenburg 2002.

WÜHRER 2000

B. WÜHRER, Merowingerzeitlicher Armschmuck aus Metall. Europe médiévale 2, Montagnac 2000. 
ZELLER 1988

K.W. Zeller, Tracht, Bewaffnung und Schmuck. In: H. DannHeImer, H. Dopsch (Hrsg.), Die Bajuwaren: Von Severin bis Tassilo 488-788 (Ausstellungskatalog der gemeinsamen Landesausstellung des Freistaates Bayern und des Landes Salzburg, Rosenbeim/Bayern, Mattsee/Salzburg, 19. Mai bis 6. November 1988). München - Salzburg 1988, 237-248.

Thomas Koch Waldner Deutsches Bergbau-Museum Bochum Am Bergbaumuseum 31 44791 Bochum Deutschland thomas.koch-waldner@bergbaumuseum.de (iD) orcid.org/0000-0002-5695-364X 